ALEA, Lat. Am. J. Probab. Math. Stat. 15, 121-149 (2018)

DOI: $10.30757 /$ ALEA.v15-06

\title{
Infinite and Giant Components in the Layers Percolation Model
}

\section{Jonathan Hermon}

University of Cambridge,

Centre for Mathematical Sciences,

Wilberforce Rd, Cambridge CB3 0WA

Cambridge, United Kingdom.

E-mail address: jonathan.hermon@statslab.cam.ac.uk

\begin{abstract}
In this work we continue the investigation launched in Feige et al. (2016) of the structural properties of the Layers model, a dependent percolation model. Given an undirected graph $G=(V, E)$ and an integer $k$, let $T_{k}(G)$ denote the random vertex-induced subgraph of $G$, generated by ordering $V$ according to Uniform $[0,1]$ i.i.d. clocks and including in $T_{k}(G)$ those vertices with at most $k-1$ of their neighbors having a faster clock. The distribution of subgraphs sampled in this manner is called the layers model with parameter $k$. The layers model has found applications in the study of $\ell$-degenerate subgraphs, the design of algorithms for the maximum independent set problem and in the study of bootstrap percolation.

We prove that every infinite locally finite tree $T$ with no leaves, satisfying that the degree of the vertices grow sub-exponentially in their distance from the root, $T_{3}(T)$ a.s. has an infinite connected component. In contrast, we show that for any locally finite graph $G$, a.s. every connected component of $T_{2}(G)$ is finite.

We also consider random graphs with a given degree sequence and show that if the minimal degree is at least 3 and the maximal degree is bounded, then w.h.p. $T_{3}$ has a giant component. Finally, we also consider $\mathbb{Z}^{d}$ and show that if $d$ is sufficiently large, then a.s. $T_{4}\left(\mathbb{Z}^{d}\right)$ contains an infinite cluster.
\end{abstract}

\section{Introduction}

Consider the following percolation model. Given a graph $G=(V, E)$, every vertex $v$ of $G$ selects independently at random an "age" $X_{v}$ from the uniform distribution Uniform $[0,1]$. For any $k \in \mathbb{N}$, define $L_{k}(G)$ to be the set of those vertices that have exactly $k-1$ younger neighbors. For an integer $k \geq 1$, we call

Received by the editors November 7th, 2016; accepted May 21th, 2017.

2010 Mathematics Subject Classification. 82B43, 05C80, 60K35.

Key words and phrases. Layers model, dependent percolation, random graphs, EIT.

Research supported by EPSRC grant EP/L018896/1. 
$L_{k}(G)$ the $k$ th layer of $G$. The union of the first $k$ layers is denoted by $T_{k}(G):=$ $\bigcup_{i=1}^{k} L_{i}(G)$. By a slight abuse of notation we refer to the subgraph induced on $T_{k}$ also by $T_{k}$, and omit $G$ when clear from context.

Note that if a vertex $v$ has $m$ neighbors in $G$, then for any $1 \leq i \leq m+1$ we have that $v \in L_{i}(G)$ with probability $\frac{1}{m+1}$. However, these events are not independent for different vertices of distance at most 2. As standard in percolation models, we say that $v \in V$ is open (closed) if $v \in T_{k}(G)$ (respectively, $v \notin T_{k}(G)$ ), where $k$ is clear from context.

The above procedure for sampling vertices from a graph has several useful properties which were exploited in the design of algorithms for finding large independent sets in graphs (e.g. Feige and Reichman, 2015) and in the study of contagious sets for bootstrap percolation (e.g. Reichman, 2012). For a list of algorithmic applications of the layers model see the related work section in Feige et al. (2016). In Feige et al. (2016) the treewidth and the size of the largest connected component of $T_{3}(G)$ and $T_{2}(G)$ were analyzed for various graphs.

In this paper we establish parallel versions of the aforementioned results from Feige et al. (2016) for infinite graphs. We also generalize a theorem in Feige et al. (2016) concerning random 3-regular graphs to random graphs with more general degree sequences (see Theorem 1.6).

We denote by $T=(V, E, o)$ a rooted-tree with root $o$. This is simply a tree with some distinct vertex, denoted by $o$. The $r$ th level of $T$, denoted by $\ell_{r}(T)$, is the collection of vertices of $T$ which are at distance $r$ from $o$. We denote the degree of $v \in V$ by $d_{v}$. In Section 2 we study $T_{3}$ on infinite trees and prove the following Theorem.

Theorem 1.1. Let $T=(V, E, o)$ be a rooted-tree of minimal degree at least 3. If there exist $C>0$ and $1 \leq a<\sqrt{4 / 3}$, such that $\max _{v \in \ell_{r}(T)} d_{v} \leq C a^{r}$ for all $r \geq 0$, then $T_{3}(T)$ has an infinite cluster a.s..

Remark 1.2. As the following example demonstrates, the degree growth condition in Theorem 1 is necessary, up to the value of $a$. Denote $a_{n}:=2^{2^{2^{n}}}$. Consider a spherically symmetric tree $T$ rooted at $o$, in which every $v \in \ell_{r}(T)$ has degree $3-1_{r=0}$ if $r \notin\left\{a_{n}: n \in \mathbb{N}\right\}$ and otherwise every $v \in \ell_{r}(T)$ has degree $\left|\ell_{r}(T)\right|+1$. Then for every $n \in \mathbb{N}$ we have that $\left|\ell_{a_{n}}(T)\right|=2^{\sum_{i=1}^{n} a_{i}}=2^{a_{n}(1+o(1))}$. Let $A_{n}$ be the event that $\ell_{a_{n}}(T) \cap T_{3}=\emptyset$. By the Borel-Cantelli Lemma a.s. infinitely many of the events $A_{n}$ occur, which implies that there are no infinite clusters in $T_{3}$.

It was shown in Feige et al. (2016) that for a bounded degree finite graph $G$ of size $n$, the largest connected component of $T_{2}(G)$ is w.h.p. ${ }^{1}$ of size at most $O(\log n)$. In Section 3 we prove an analogous result in the infinite setup, while dropping the assumption that $G$ is of bounded degree.

Theorem 1.3. Let $G=(V, E)$ be a locally finite graph with a countable vertex set $V$. Then,

$$
\mathrm{P}\left[T_{2}(G) \text { has an infinite connected component }\right]=0 \text {. }
$$

The only infinite graph considered in Feige et al. (2016) is $\mathbb{Z}^{2}$, for which it was shown that $T_{4}\left(\mathbb{Z}^{2}\right)$ a.s. has a unique infinite connected component (which we also call an infinite cluster).

In Section 4 we consider $T_{4}\left(\mathbb{Z}^{d}\right)$ for $d>2$ and prove the following theorem.

\footnotetext{
${ }^{1}$ With high probability - that is, with probability tending to 1 as $n \rightarrow \infty$.
} 
Theorem 1.4. $T_{4}\left(\mathbb{Z}^{d}\right)$ has an infinite cluster a.s., for all sufficiently large $d$.

In fact, we prove a stronger assertion than that of Theorem 1.4. Theorem 4.1 asserts that $T_{4}\left(\mathbb{Z}^{d}\right)$ contains an open infinite monotone path ${ }^{2}$ a.s., for all sufficiently large $d$. We expect Theorem 1.4 to hold for all $d$, however it seems that even with a more careful analysis, the argument in the proof of Theorem 1.4 cannot be used to prove this, because of the restriction of the argument to monotone paths.

Question 1.5. Does $T_{3}\left(\mathbb{Z}^{d}\right)$ a.s. contain a unique infinite connected component for all $d \geq 1$ ?

In Feige et al. (2016) it was shown that for random 3-regular graphs, w.h.p. ${ }^{3}$ $T_{3}$ contains a giant component ${ }^{4}$. In Section 5 we generalize the aforementioned result by considering random graphs with more general degree sequences. Denote $[n]:=\{1, \ldots, n\}$.

Theorem 1.6. Let $d \geq 3$. Let $G=([n], E)$ be a random graph chosen from the uniform distribution over all labeled graphs satisfying that the degree of vertex $i$ equals $d_{i}$ for some sequence of numbers $\left(d_{i}: 1 \leq i \leq n\right)$ such that $\sum_{i} d_{i}$ is even and $3 \leq d_{i} \leq d$, for all $i \in[n]$. Then there exists a constant $\alpha=\alpha(d)$ such that

$$
\mathrm{P}\left[T_{3}(G) \text { contains a connected component of size at least } \alpha n\right]=1-\exp (-\Omega(n)) \text {, }
$$

where the probability is taken jointly over the choice of $G$ and of the ages of the vertices.

Note that in Theorems 1.1 and 1.3 we do not assume that the graphs are of bounded degree. Similarly, in Theorem 1.6 we allow fairly general degree sequences. This aspect of Theorems 1.1, 1.3 and 1.6 is interesting, since a-priori, it is not obvious how to construct a canonical site percolation process, that has the same marginal probabilities as the Layers model, which exhibits such behaviors.

It is interesting to note that " 3 " is the critical value for the Layers model, both in the setup of Theorem 1.1 and of Theorem 1.6 (in the sense that $T_{3}$ has an infinite cluster (a.s. in the infinite setting) or a giant component (w.h.p. in the finite setting)). This is not a coincidence. Our proof of Theorem 1.6 rely heavily on the analysis of the layers model on trees. It is well-known that, in some sense, the random graphs considered in Theorem 1.6, locally look like trees. Thus it is natural to interpret the coincidence of the critical value " 3 " in Theorems 1.1 and 1.6 as an instance in which the critical parameter is a "local property". Such locality is conjectured to hold in greater generality for Bernoulli (independent) Percolation Benjamini et al. (2011) (the results in Benjamini et al., 2011 cover in particular the case of large girth expanders).

1.1. An overview of our techniques. We now present a short informal discussion which summarizes the main ideas in this paper.

Let $G=(V, E)$ be a graph and $k \in \mathbb{N}$. Let $Y_{v}$ be the indicator of the event that vertex $v$ belongs to $T_{k}(G)$. Clearly, if $A \subset V$ and for any distinct $v, u \in A$ we have that $u$ and $v$ are not neighbors and do not have a common neighbor, then $\left(Y_{v}\right)_{v \in A}$

\footnotetext{
${ }^{2}$ A path w.r.t. an oriented copy of $\mathbb{Z}^{d}$.

${ }^{3}$ Over the joint distribution of the random 3-regular graph $H$ and $T_{3}(H)$.

${ }^{4}$ A giant component is a connected component of size $\Omega(n)$
} 
are independent. However, if there exist $u, v \in A$ which are neighbors or have a common neighbor, then typically $\left(Y_{v}\right)_{v \in A}$ are not independent.

It is hard to analyze the possible global effects of such dependencies. To avoid this, it is useful to consider a family of paths in $G, \Gamma$, and studying the first and second moments of the number (or more generally, the "mass" with respect to some measure on $\Gamma$ ) of paths in $\Gamma$ which are contained in $T_{k}(G)$. This requires one to deal only with dependencies between vertices along at most two paths.

A substantial amount of "paths" techniques were developed in the study of percolation. The most relevant ones to this work are the second moment method and the $\mathbf{E I T}^{5}$ property.

In Section 2 we study $T_{3}$ on trees. As previously described, using paths techniques, we reduce the complexity of dependencies we have to deal with. Exploiting some hidden structure allows us to control the dependencies along two paths (a similar hidden structure is exploited also in the proof of Theorem 1.4). Essentially, we show that for every tree $T$ as in Theorem 1.1 we have that $T_{3}(T)$ stochastically dominates some super-critical quasi-independent percolation process on $T$ (see (2.3) for a precise statement). Unfortunately, the situation is more involved than that and we cannot use results regarding quasi-independent percolation as a black box. Instead, we perform a weighted second moment calculation.

The study of quasi-independent percolation on trees ${ }^{6}$ and its relations to independent percolation is due to Lyons $(1989,1992)$ (see also Sections 5.3-5.4 in Lyons and Peres, 2016).

The EIT method was introduced in Cox and Durrett (1983), where it was exploited for showing that the critical value for oriented independent bond percolation on $\mathbb{Z}^{d}$ is $d^{-1}+O\left(d^{-3}\right)$. The method was further extended in Benjamini et al. (1998). The novelty of our use of the EIT method is that we apply it in a dependent setup using an auxiliary Markov chain which represents the different type of dependencies between two vertices. This idea can be utilized in some other dependent percolation models in which there is a bounded range of dependencies.

1.2. Notation and terminology. Given a graph $G=(V, E)$, the connected component containing $v$ is denoted by $C(v)$. We define the length of a path $\gamma$ as the number of edges it contains and denote it by $|\gamma|$. By abuse of notation we often identify a path $\gamma=\left(\gamma_{1}, \gamma_{2}, \ldots, \gamma_{k}\right)$ with the set $\left\{\gamma_{1}, \gamma_{2}, \ldots, \gamma_{k}\right\}$. Throughout, we denote the $i$ th vertex in $\gamma$ by $\gamma_{i}(1 \leq i \leq|\gamma|+1)$. We say that the path $\gamma$ is simple (or self-avoiding) if $\gamma_{i} \neq \gamma_{j}$, for all $i \neq j$. We denote the collection of all simple paths of length $k$ starting from $v \in V$ by $\Gamma_{v, k}$ and the collection of all such infinite simple paths by $\Gamma_{v}$.

Given a pair of vertices $u, v \in V$ their distance $d_{G}(u, v)$ is defined as the length of the shortest path in $G$ starting at $u$ and ending at $v^{7}$. For $A, B \subseteq V$, the distance of $A$ from $B$ in $G$ is defined to be $d_{G}(A, B):=\inf \left\{d_{G}(a, b): a \in A, b \in B\right\}$. When $A$ and $B$ are not disjoint, we define $d_{G}(A, B)=0$. When the underlining graph is clear from context, we simply write $d(A, B)$ and $d(u, v)$. When $A=\{u\}$ we write $d(u, B)$ instead of $d(\{u\}, B)$.

\footnotetext{
${ }^{5}$ Exponential intersection tail.

${ }^{6}$ Also known as quasi-Bernoulli percolation.

${ }^{7}$ If $u$ and $v$ are not connected by any path, then their distance is defined to be $\infty$.
} 
For a pair of vertices $u, v$, we write $u \sim v$ if $d(u, v)=1$ and say that $u$ is adjacent to (or a neighbor of) $v$. We say that a graph $G=(V, E)$ is locally finite if $d_{v}<\infty$ for all $v \in V$. We say that a vertex $v$ is a neighbor of a set $A$ and write $v \sim A$ (or of a path $\gamma$ and write $v \sim \gamma$ ), if $d(v, A)=1$ (respectively, $d(v, \gamma)=1$ ). That is, if $v \notin A$ (respectively, $v \notin \gamma$ ) and there is some $u \in A$ (respectively, $u \in \gamma$ ) such that $v \sim u$. If $d(v, A)>1$ we write $v \nsim A$. When $A=\{u\}$ we write $v \nsim u$.

We often abbreviate and write w.p. for "with probability". We call a random variable which takes the value 1 w.p. $p$ and the value 0 w.p. $1-p$ a $\operatorname{Bernoulli}(p)$ random variable. We denote the indicator of an event $A$ by $1_{A}$.

Several results that are used in the paper are quoted in the appendix (Section 6). All of these results are standard, with the exception of Theorem 6.4.

\section{Infinite trees}

In this section we study $T_{3}$ on infinite trees and prove Theorem 1.1. Fix some simple path $\gamma$ of length $2 k-1$ for some $k \geq 1$. We shall dominate the restriction of $T_{3}$ to $\gamma$ using an auxiliary percolation process which is amenable to relatively neat analysis. For this purpose we partition $\gamma$ into successive pairs, $\left\{\gamma_{2 i-1}, \gamma_{2 i}\right\}$, $i=1,2, \ldots, k$. For each $i$ we define a certain "good" event $A_{i}(\gamma)$, depending only on the ages of $\gamma_{2 i-1}, \gamma_{2 i}$ and of that of their neighbors which do not lie in $\gamma$. The motivation behind the definitions shall be explain soon.

Let $T=(V, E, o)$ be a locally finite rooted tree. For any $k \in \mathbb{N}$, let $\Gamma_{2 k-1}$ be the collection of all self-avoiding paths of length $2 k-1$ starting at $o$.

Definition 2.1. Let $T=(V, E, o)$ be a locally finite rooted tree. For any $v \in$ $V \backslash\{o\}$ the parent of $v$, denoted by $\bar{v}$, is the unique vertex such that $\bar{v} \sim v$ and $d(o, \bar{v})=d(o, v)-1$. Let $\ell_{r}:=\{v \in V: d(o, v)=r\}, r \geq 0$. Let $\gamma \in \Gamma_{2 k-1}$. We define $N_{i}(\gamma):=\left\{u \sim \gamma_{i}: u \notin \gamma\right\}$ to be the set of neighbors of $\gamma_{i}$ which do not lie in $\gamma$. For every $i \in[k]$ and $j \in[2 k]$ we define

$$
\begin{aligned}
M_{2 i-1}(\gamma) & :=\left|\left\{u \in N_{2 i-1}(\gamma) \cup\left\{\gamma_{2 i}\right\}: X_{\gamma_{2 i-1}}>X_{u}\right\}\right|, \\
M_{2 i}(\gamma) & :=\left|\left\{u \in N_{2 i}(\gamma) \cup\left\{\gamma_{2 i-1}\right\}: X_{\gamma_{2 i}}>X_{u}\right\}\right|, \\
K_{j}(\gamma) & :=\left|\left\{u \in N_{j}(\gamma): X_{\gamma_{j}}>X_{u}\right\}\right| .
\end{aligned}
$$

For all $1<i<k$, let $A_{i}(\gamma)$ be the event that $\max \left(M_{2 i-1}(\gamma), M_{2 i}(\gamma)\right) \leq 1$. Let $A_{1}(\gamma)$ (resp. $\left.A_{k}(\gamma)\right)$ be the event that $K_{1}(\gamma) \leq 2, K_{2}(\gamma) \leq 1\left(\operatorname{resp} . K_{2 k}(\gamma) \leq 2\right.$, $\left.K_{2 k-1}(\gamma) \leq 1\right)$. We say that a path $\gamma \in \Gamma_{2 k-1}$ is good, if the event $A_{\gamma}:=\bigcap_{i=1}^{k} A_{i}(\gamma)$ occurs. For any path $\gamma \in \Gamma_{k}$ we set

$$
w(\gamma):=\frac{1}{d_{o}} \prod_{i=2}^{2 k-1} \frac{1}{d_{\gamma_{i}}-1} \text { and } Y_{\gamma}:=\frac{w(\gamma) 1_{A_{\gamma}}}{\mathrm{P}\left[A_{\gamma}\right]} .
$$

Finally, we say that $o$ is $k$-good, if $\sum_{\gamma \in \Gamma_{2 k-1}} Y_{\gamma} \geq \frac{1}{2}$.

Note that by construction, a good path must be contained in $T_{3}$. The idea behind the definition of a good path is that for a fixed $\gamma \in \Gamma_{2 k-1}$, the events $A_{1}(\gamma), A_{2}(\gamma), \ldots, A_{k}(\gamma)$ are mutually independent, as they depend on ages of disjoint sets of vertices (since $T$ is a tree). This avoids dealing with the accumulation of dependencies, which may occur when working with the indicators of the vertices along a certain path belonging to $T_{3}$. 
Fix some path $\gamma \in \Gamma_{2 k-1}$. The analysis would have been much simpler if instead of considering $A_{1}(\gamma), A_{2}(\gamma), \ldots, A_{k}(\gamma)$ we could work with the events $I_{1}(\gamma), \ldots, I_{2 k}$, where $I_{i}(\gamma)$ is the event that $\gamma_{i}$ is younger than all of its neighbors which do not lie in $\gamma$. The events $I_{1}(\gamma), \ldots, I_{2 k}(\gamma)$ are independent and for all $i$ we have that $I_{i}(\gamma)$ is contained in the event that $\gamma_{i} \in T_{3}$. However, the $I_{i}(\gamma)$ 's give rise to a critical percolation process which does not suffice for our purposes.

We now describe a growth condition that shall be assumed throughout the section. Whenever the minimal degree of $T$ is strictly greater than 2 , the condition takes a simple form and coincides with the growth condition appearing in Theorem 1.1. In particular, our analysis implies the assertion of Theorem 1.1, but allows us to deal also with vertices of degree 2 .

For any $v \in T$ let $\gamma^{(v)}$ be the unique self-avoiding path in $T$ which starts at $o$ and ends at $v$. We define $q_{v}:=\left|\left\{1<i<\left\lfloor\frac{d(v, o)+1}{2}\right\rfloor: \max \left(d_{\gamma_{2 i-1}^{(v)}}, d_{\gamma_{2 i}^{(v)}}\right)>2\right\}\right|$. Consider the following condition. There exist $C>0$ and $1 \leq a<4 / 3$, such that

$$
d_{v}-1 \leq C a^{q_{v}} d_{o} \text {, for all } v \in V .
$$

Definition 2.2. For any distinct paths $\gamma, \gamma^{\prime} \in \Gamma_{2 k-1}$, let $\gamma \wedge \gamma^{\prime}$ be the furthest vertex from $o$ belonging to both paths. Let $\left|\gamma \wedge \gamma^{\prime}\right|:=d\left(o, \gamma \wedge \gamma^{\prime}\right)+1\left(\right.$ so $\gamma_{\left|\gamma \wedge \gamma^{\prime}\right|}=$ $\left.\gamma \wedge \gamma^{\prime}\right)$. Finally, we define

$$
\gamma \cap \gamma^{\prime}:=\left(\gamma_{1}, \gamma_{2}, \ldots, \gamma_{\left|\gamma \wedge \gamma^{\prime}\right|}\right) \in \Gamma_{\left|\gamma \wedge \gamma^{\prime}\right|} .
$$

Essentially, we will show that the percolation process defined via the good-paths is a quasi-(independent) Bernoulli percolation process (see Lyons and Peres, 2016 section 5.4 for a precise definition and details). More precisely, we show that there exists an absolute constant $M>0$ such that

$$
\begin{aligned}
& \mathrm{P}\left[A_{\gamma} \cap A_{\gamma^{\prime}}\right] \geq \frac{\mathrm{P}\left[A_{\gamma}\right] \mathrm{P}\left[A_{\gamma^{\prime}}\right]}{M \mathrm{P}\left[A_{\gamma \cap \gamma^{\prime}}\right]}, \text { for all } \gamma, \gamma^{\prime} \in \Gamma_{2 k-1} \text { such that }\left|\gamma \wedge \gamma^{\prime}\right| \text { is even, } \\
& \mathrm{P}\left[A_{\gamma} \cap A_{\gamma^{\prime}}\right] \geq \frac{\mathrm{P}\left[A_{\gamma}\right] \mathrm{P}\left[A_{\gamma^{\prime}}\right] d_{\left|\gamma \wedge \gamma^{\prime}\right|}}{M \mathrm{P}\left[A_{\left(\gamma \cap \gamma^{\prime} \backslash\left\{\gamma \wedge \gamma^{\prime}\right\}\right)}\right]}, \text { for all } \gamma, \gamma^{\prime} \in \Gamma_{2 k-1} \text { such that }\left|\gamma \wedge \gamma^{\prime}\right| \text { is odd. }
\end{aligned}
$$

Although (2.3) bears a resemblance to the condition which defines a quasi-Bernoulli percolation process, because the $A_{i}(\gamma)$ 's are defined in terms of pairs of vertices, we cannot use results concerning quasi-Bernoulli percolation as a black box.

Nevertheless, such results are useful in terms of intuition. Moreover, since the main tool utilized in the development of the theory of quasi-Bernoulli percolation is the second moment method, it is only natural to utilize the same technique when attempting to transform our intuition into a rigorous proof.

As can be seen from (2.5) and (2.6) below, in conjunction with the independence of the events $A_{1}(\gamma), A_{2}(\gamma), \ldots, A_{k}(\gamma)$, for each fixed $\gamma \in \Gamma_{2 k-1}$, the marginal probabilities of the $A_{\gamma}$ 's would have implied super-criticality for independent site percolation, at least when $T$ is of bounded degree. Thus, if we actually had a quasiBernoulli percolation process in hand, then Theorems 5.19 and 5.24 in Lyons and Peres (2016) (originally from Lyons, 1989,1992$)$ would have implied that $T_{3}(T)$ is super-critical (at least when $T$ is of bounded degree).

Theorem 2.3. Let $T=(V, E, o)$ be a rooted tree with no leaves. Assume that condition (2.2) holds for some $C>0$ and $1<a<4 / 3$. Then there exists some constant $c_{1}>0$, such that $o$ is $k$-good with probability at least $c_{1} / d_{o}$, for all $k>1$. 
Proof: Fix some $k>1$. For a path $\gamma \in \Gamma_{2 k-1}$ let $Y_{\gamma}$ be as in (2.1). Then $\mathbf{E}\left(Y_{\gamma}\right)=$ $w(\gamma)$, for all $\gamma \in \Gamma_{2 k-1}$. We define $Z_{k}:=\sum_{\gamma \in \Gamma_{2 k-1}} Y_{\gamma}$. By the definition of $w(\gamma)$ we have that $\sum_{\gamma \in \Gamma_{2 k-1}} w(\gamma)=1$. Hence, $\mathbf{E}\left[Z_{k}\right]=1$. By the Paley-Zygmund inequality,

$$
\mathrm{P}(o \text { is } k \text {-good })=\mathrm{P}\left(Z_{k}>1 / 2\right) \geq \frac{1}{4 \mathbf{E}\left[Z_{k}^{2}\right]} .
$$

Thus our task is to estimate $\mathbf{E}\left[Z_{k}^{2}\right]$ from above. Note that

$$
\mathbf{E}\left[Z_{k}^{2}\right]=\sum_{\gamma \in \Gamma_{2 k-1}} \sum_{\gamma^{\prime} \in \Gamma_{2 k-1}} \mathbf{E}\left[Y_{\gamma} Y_{\gamma^{\prime}}\right]=\sum_{\gamma \in \Gamma_{2 k-1}} \sum_{\gamma^{\prime} \in \Gamma_{2 k-1}} w(\gamma) w\left(\gamma^{\prime}\right) \frac{\mathrm{P}\left[A_{\gamma} \cap A_{\gamma^{\prime}}\right]}{\mathrm{P}\left[A_{\gamma}\right] \mathrm{P}\left[A_{\gamma^{\prime}}\right]} .
$$

Let $\gamma \in \Gamma_{2 k-1}$. We now calculate $\mathrm{P}\left[A_{\gamma}\right]$. Since we are considering a tree, by construction, the events $A_{1}(\gamma), A_{2}(\gamma), \ldots, A_{k}(\gamma)$ depend on ages of disjoint sets of

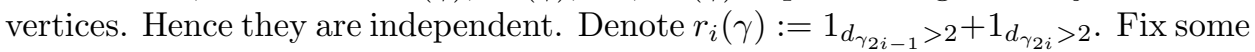
$i \neq 1, k$. There are three cases in which we can have $\left\{M_{2 i-1}(\gamma) \leq 1, M_{2 i}(\gamma) \leq 1\right\}$ :

Case 1: $K_{2 i-1}(\gamma)=0=K_{2 i}(\gamma)$ - That is, both $\gamma_{2 i-1}$ and $\gamma_{2 i}$ are younger than all of their neighbors (resp.) which do not lie in $\gamma$. Because $N_{2 i-1}(\gamma) \cap N_{2 i}(\gamma)=\emptyset$, we have that $K_{2 i-1}(\gamma)$ and $K_{2 i}(\gamma)$ are independent and so the probability they both equal 0 is $\frac{1}{\left(d_{\gamma_{2 i-1}}-1\right)\left(d_{\gamma_{2 i}}-1\right)}$.

Case 2: $K_{2 i-1}(\gamma)=1, X_{\gamma_{2 i}}>X_{\gamma_{2 i-1}}, K_{2 i}(\gamma)=0$ - That is, $\gamma_{2 i}$ is younger than all of its neighbors which do not lie in $\gamma$, but older than $\gamma_{2 i-1}$, while $\gamma_{2 i-1}$ has exactly one younger neighbor not lying in $\gamma$. There are $d_{\gamma_{2 i-1}}-2$ possibilities for the identity of the youngest member of $N_{2 i-1}(\gamma)$, which in fact has to be the youngest in $N_{2 i-1}(\gamma) \cup N_{2 i}(\gamma) \cup\left\{\gamma_{2 i-1}, \gamma_{2 i}\right\}$ (contributing a $\frac{d_{\gamma_{2 i-1}}-2}{d_{\gamma_{2 i-1}}+d_{\gamma_{2 i}}-2}$ factor), while $\gamma_{2 i-1}$ has to be the second youngest in this set (contributing a $\frac{1}{d_{\gamma_{2 i-1}}+d_{\gamma_{2 i}}-3}$ factor). Conditioned on that, the distribution of the relative order between $N_{2 i}(\gamma) \cup\left\{\gamma_{2 i}\right\}$ is still uniform over all orderings, and so the conditional probability that $\gamma_{2 i}$ is the youngest in this set is $\frac{1}{d_{\gamma_{2 i}}-1}$. Overall, the probability of Case 2 is $\frac{d_{\gamma_{2 i-1}}-2}{\left(d_{\gamma_{2 i-1}}+d_{\gamma_{2 i}}-2\right)\left(d_{\gamma_{2 i-1}}+d_{\gamma_{2 i}}-3\right)\left(d_{\gamma_{2 i}}-1\right)}$.

Case 3: $K_{2 i-1}(\gamma)=0, X_{\gamma_{2 i}}<X_{\gamma_{2 i-1}}, K_{2 i}(\gamma)=1$ (same as Case 2, with the roles of $2 i-1$ and $2 i$ exchanged), which has probability $\frac{d_{\gamma_{2 i}}-2}{\left(d_{\gamma_{2 i-1}}+d_{\gamma_{2 i}}-2\right)\left(d_{\gamma_{2 i-1}}+d_{\gamma_{2 i}}-3\right)\left(d_{\gamma_{2 i-1}}-1\right)}$.

Let $f(x, y):=\frac{(x-1)(x-2)+(y-1)(y-2)}{(x+y-2)(x+y-3)}$. We get that:

$$
\mathrm{P}\left[A_{i}(\gamma)\right]= \begin{cases}\frac{1+f\left(d_{\gamma_{2 i-1}}, d_{\gamma_{2 i}}\right)}{\left(d_{\gamma_{2 i}-1}-1\right)\left(d_{\gamma_{2 i}}-1\right)}, & 1<i<k, \\ \frac{2}{d_{o}\left(d_{\gamma_{2}}-1\right)}, & i=1, \\ \frac{2}{\left(d_{\gamma_{2 k-1}}-1\right) d_{\gamma_{2 k}}}, & i=k .\end{cases}
$$

Claim 2.4. The function $f(x, y)=\frac{(x-1)(x-2)+(y-1)(y-2)}{(x+y-2)(x+y-3)}$ attains its minimum in the domain $D:=\left\{(x, y) \in \mathbb{R}^{2}: x, y \geq 3\right\}$ at $(x, y)=(3,3)$ and $f(3,3)=1 / 3$. Whereas $f(x, 2)=1-\frac{2}{x}$ and $f(2, y)=1-\frac{2}{y}$.

Proof: Observe that since $(x+y)^{2} \leq 2 x^{2}+2 y^{2}$, we have that

$$
\limsup _{r \rightarrow \infty} \sup _{(x, y) \in D: x+y \geq r} f(x, y)=\limsup _{r \rightarrow \infty} \sup _{(x, y) \in D: x+y \geq r} \frac{x^{2}+y^{2}}{(x+y)^{2}}=1 / 2
$$


Since $f(x, y)<1 / 3<1 / 2$ this means that $f$ has to attain a global minimum in the domain $D$. We now verify that there is no solution to $\partial_{x} f(x, y)=0=\partial_{y} f(x, y)$ in $D$. Indeed, $\partial_{x} f(x, y)=\frac{(y-1)\left(x^{2}-2 x-y^{2}+3 y-1\right)}{(x+y-2)^{2}(x+y-3)^{2}}$ whose only root which is greater or equal to 3 is $x_{0}(y)=1+\sqrt{(y-1)(y-2)}$. By symmetry, if $\partial_{y} f(x, y)=0$, then $y=y_{0}(x)=1+\sqrt{(x-1)(x-2)}$. Hence if $\partial_{x} f(x, y)=0=\partial_{y} f(x, y)$, we must have that

$$
x=1+\sqrt{(y-1)(y-2)}=1+\sqrt{(\sqrt{(x-1)(x-2)})(\sqrt{(x-1)(x-2)}-1)},
$$

which implies that $x=\sqrt{(x-1)(x-2)}$, a contradiction! Thus if the minimum is attained at $(x, y)$, we must have that either $x=3$ or $y=3$ (since the minimum must be attained at the boundary of the domain). By symmetry, we may assume that $y=3$. Minimizing $f(x, 3)$ over $x \geq 3$, it is not hard to verify that the minimum is attained at $x=3$.

Note that if $1<i<k$ and $\max \left(d_{\gamma_{2 i-1}}, d_{\gamma_{2 i}}\right)=2$ (i.e. $r_{i}(\gamma)=0$ ) we get that $\mathrm{P}\left[A_{i}(\gamma)\right]=1$, while if $r_{i}(\gamma)>0$ (i.e. $\max \left(d_{\gamma_{2 i-1}}, d_{\gamma_{2 i}}\right)>2$ ) then by (2.5) and Claim 2.4

$$
\mathrm{P}\left[A_{i}(\gamma)\right] \geq \frac{4}{3\left(d_{\gamma_{2 i-1}}-1\right)\left(d_{\gamma_{2 i}}-1\right)}, \text { for all } 1<i<k \text { such that } r_{i}(\gamma)>0 .
$$

We now turn to the task of finding an upper bound on $\mathrm{P}\left[A_{\gamma} \cap A_{\gamma^{\prime}}\right]$ for $\gamma, \gamma^{\prime} \in$ $\Gamma_{2 k-1}$. Fix some distinct $\gamma, \gamma^{\prime} \in \Gamma_{k}$. If $\left|\gamma \wedge \gamma^{\prime}\right| \in\{2 i-1,2 i\}$, we define $B_{\gamma, \gamma^{\prime}}$ to be the event that for $j \in\{2 i-1,2 i\}$ we have that $\left|\left\{u: X_{\gamma_{j}}>X_{u}, u \sim \gamma_{j}, u \notin \gamma \cup \gamma^{\prime}\right\}\right| \leq 1$ and that also $\left|\left\{u: X_{\gamma_{j}^{\prime}}>X_{u}, u \sim \gamma_{j}^{\prime}, u \notin \gamma \cup \gamma^{\prime}\right\}\right| \leq 1$.

Note that $A_{i}(\gamma) \cap A_{i}\left(\gamma^{\prime}\right) \subset B_{\gamma, \gamma^{\prime}}$. Thus if $\left|\gamma \wedge \gamma^{\prime}\right|=j<2 k$, then

$$
A_{\gamma} \cap A_{\gamma^{\prime}} \subset\left(\bigcap_{i \in[k] \backslash\{\lfloor j / 2\rfloor\}} A_{i}(\gamma)\right) \cap B_{\gamma, \gamma^{\prime}} \cap\left(\bigcap_{i=\lfloor j / 2\rfloor+1}^{k} A_{i}\left(\gamma^{\prime}\right)\right),
$$

where if $\left|\gamma \wedge \gamma^{\prime}\right|=2 k-2$, the rightmost intersection does not appear. Moreover, from the definition of $B_{\gamma, \gamma^{\prime}}$, we have that the $2 k-\lfloor j / 2\rfloor$ events appearing in the right hand side of (2.7) are jointly independent, as they depend on ages of disjoint sets of vertices. Consequently, if $\left|\gamma \wedge \gamma^{\prime}\right|=j<2 k$ then

$$
\frac{\mathrm{P}\left[A_{\gamma} \cap A_{\gamma^{\prime}}\right]}{\mathrm{P}\left[A_{\gamma}\right] \mathrm{P}\left[A_{\gamma^{\prime}}\right]} \leq \frac{\mathrm{P}\left[B_{\gamma, \gamma^{\prime}}\right]}{\mathrm{P}\left[A_{\lfloor j / 2\rfloor}(\gamma)\right] \mathrm{P}\left[A_{\lfloor j / 2\rfloor}\left(\gamma^{\prime}\right)\right] \prod_{0 \leq i<\lfloor j / 2\rfloor} \mathrm{P}\left[A_{i}(\gamma)\right]}
$$

We now calculate $\mathrm{P}\left[B_{\gamma, \gamma^{\prime}}\right]$. Assume that $\left|\gamma \wedge \gamma^{\prime}\right| \in\{2 i-1,2 i\}$. If $\left|\gamma \wedge \gamma^{\prime}\right|=2 i$, then $B_{\gamma, \gamma^{\prime}}$ consists of two independent events, defined in terms of $\gamma_{2 i-1}$ and $\gamma_{2 i}$, respectively. Similarly, if $\left|\gamma \wedge \gamma^{\prime}\right|=2 i-1$, then $B_{\gamma, \gamma^{\prime}}$ consists of three independent events, defined in terms of $\gamma_{2 i-1}, \gamma_{2 i}$ and $\gamma_{2 i}^{\prime}$, respectively. A simple calculation yields that:

$$
\mathrm{P}\left[B_{\gamma, \gamma^{\prime}}\right]= \begin{cases}\frac{8}{\left(d_{\gamma_{2 i}}-1\right)\left(d_{\gamma_{2 i}^{\prime}}-1\right)\left(d_{\gamma_{2 i-1}}-2\right)}, & \left|\gamma \wedge \gamma^{\prime}\right|=2 i-1, i \notin\{1,2 k-1\}, \\ 4\left(d_{\gamma_{2 i}}-2\right)^{-1}\left(d_{\gamma_{2 i-1}}-1\right)^{-1}, & \left|\gamma \wedge \gamma^{\prime}\right|=2 i, i \notin\{1,2 k-1\}, \\ 8\left(d_{o}-1\right)^{-1}\left(d_{\gamma_{2}}-1\right)^{-1}\left(d_{\gamma_{2}^{\prime}}-1\right)^{-1}, & \left|\gamma \wedge \gamma^{\prime}\right|=1, \\ 4\left(d_{o}\left(d_{\gamma_{2}}-2\right)\right)^{-1}, & \left|\gamma \wedge \gamma^{\prime}\right|=2, \\ 8\left(\left(d_{\gamma_{2 k-1}}-2\right) d_{\gamma_{2 k}} d_{\gamma_{2 k}^{\prime}}\right)^{-1}, & \left|\gamma \wedge \gamma^{\prime}\right|=2 k-1 .\end{cases}
$$


Plugging this and (2.5)-(2.6) in (2.8) yields that if $\left|\gamma \wedge \gamma^{\prime}\right|=j<2 k$, then

$$
\frac{\mathrm{P}\left[A_{\gamma} \cap A_{\gamma^{\prime}}\right]}{\mathrm{P}\left[A_{\gamma}\right] \mathrm{P}\left[A_{\gamma^{\prime}}\right]} \leq 8 \cdot(3 / 4)^{\left|\left\{1<m<\lfloor j / 2\rfloor: r_{m}(\gamma) \neq 0\right\}\right|}\left(d_{o} / 2\right) \prod_{1 \leq i \leq j}\left(d_{\gamma_{i}}-1\right) .
$$

Denote $W(v):=\sum_{\tilde{\gamma} \in \Gamma_{k}: v \in \tilde{\gamma}} w(\tilde{\gamma})$ for every $v \in V$ such that $d(v, o) \leq 2 k-1$. Note that

$$
d_{o} \prod_{2 \leq i \leq j-1}\left(d_{\gamma_{i}}-1\right)=\frac{1}{W\left(\gamma_{j}\right)} \text {, for all } \gamma \in \Gamma_{2 k-1} \text { and } j \leq 2 k .
$$

Let $1 \leq a<4 / 3$ and $C>0$ be as in condition (2.2). Then $\alpha:=\frac{3 a}{4}<1$. Recall that from the definition of $q_{v}$, from condition (2.2), $q_{\gamma_{j}}=\left|\left\{1<m<\lfloor j / 2\rfloor: r_{m}(\gamma) \neq 0\right\}\right|$. By condition (2.2), $d_{\gamma_{j}}-1 \leq C a^{q_{\gamma_{j}}}$. Hence

$$
4 \cdot\left(d_{\gamma_{j}}-1\right)(3 / 4)^{q_{\gamma_{j}}} \leq C_{1} \alpha^{q_{\gamma_{j}}} d_{o},
$$

This, together with (2.10)-(2.11), implies that

$$
\frac{\mathrm{P}\left[A_{\gamma} \cap A_{\gamma^{\prime}}\right]}{\mathrm{P}\left[A_{\gamma}\right] \mathrm{P}\left[A_{\gamma^{\prime}}\right]} \leq \frac{C_{1} \alpha^{q_{\gamma_{j}}} d_{o}}{W\left(\gamma_{j}\right)} \text {, for all } \gamma, \gamma^{\prime} \in \Gamma_{2 k-1} \text { such that }\left|\gamma \wedge \gamma^{\prime}\right|=j<2 k .
$$

It is easy to verify that $\frac{\mathrm{P}\left[A_{\gamma} \cap A_{\gamma^{\prime}}\right]}{\mathrm{P}\left[A_{\gamma}\right] \mathrm{P}\left[A_{\gamma^{\prime}}\right]} \leq \frac{C_{1} \alpha^{q} \gamma_{2 k} d_{o}}{W\left(\gamma_{2 k}\right)}$ also when $\gamma=\gamma^{\prime}$. Note for all $\bar{\gamma} \in \Gamma_{2 k-1}$ we have that $\sum_{\tilde{\gamma} \in \Gamma_{2 k-1}:\left|\tilde{\gamma} \wedge \bar{\gamma}^{\prime}\right|=r} w(\tilde{\gamma}) \leq W\left(\bar{\gamma}_{r}\right)$, for all $r \leq 2 k$. Hence, by (2.12) we have that:

$$
\begin{aligned}
& \sum_{\gamma^{\prime} \in \Gamma_{2 k-1}} \mathbf{E}\left[Y_{\gamma} Y_{\gamma^{\prime}}\right] \leq C_{1} d_{o} w(\gamma) \sum_{j=1}^{2 k} \sum_{\gamma^{\prime} \in \Gamma_{2 k-1}:\left|\gamma \wedge \gamma^{\prime}\right|=j} w\left(\gamma^{\prime}\right) \alpha^{q_{\gamma_{j}}} / W\left(\gamma_{j}\right) \\
& \leq C_{1} d_{o} w(\gamma)\left[2+\sum_{3 \leq j \leq 2 k: d_{\gamma_{j}}>2} \sqrt{\alpha}^{\left|\left\{3 \leq i \leq j-2: d_{\gamma_{i}}>2\right\}\right|}\right] \leq M d_{o} w(\gamma),
\end{aligned}
$$

for some constant $M>0$. Hence

$$
\mathbf{E}\left[Z_{k}^{2}\right]=\sum_{\gamma \in \Gamma_{2 k-1}} \sum_{\gamma^{\prime} \in \Gamma_{2 k-1}} \mathbf{E}\left[Y_{\gamma} Y_{\gamma^{\prime}}\right] \leq \sum_{\gamma \in \Gamma_{2 k-1}} M d_{o} w(\gamma)=M d_{o}
$$

Finally, by the Paley-Zygmund inequality, $\mathrm{P}\left(Z_{k}>1 / 2\right) \geq \frac{1}{4 M d_{o}}$.

Lemma 2.5. Let $G=(V, E)$ be an infinite connected graph. Let $\ell \in \mathbb{N}$. For any $v \in V$, let $Y_{v}$ be the indicator of the event that $v \in T_{\ell}(G)$. Then the tail $\sigma$-algebra of $\left(Y_{v}\right)_{v \in V}$ is trivial. Consequently, $\mathrm{P}\left[A_{\ell}\right] \in\{0,1\}$, where $A_{\ell}$ is the event that $T_{\ell}(G)$ has an infinite cluster.

Proof: One can readily verify that the tail $\sigma$-algebra of $\left(Y_{v}\right)_{v \in V}$ is contained in the tail $\sigma$-algebra of $\left(X_{v}\right)_{v \in V}$. Thus it is trivial by Kolmogorov's 0-1 law. It is easy to see that $A_{\ell}$ is in the tail $\sigma$-algebra of $\left(Y_{v}\right)_{v \in V}$.

Corollary 2.6. Let $T=(V, E, o)$ be a rooted tree with no leaves. Assume that condition (2.2) holds. Then $\mathrm{P}\left[\left|C_{o}\left(T_{3}\right)\right|=\infty\right] \geq c_{1} / d_{o}$, where $C_{o}\left(T_{3}\right)$ is the connected component of o in $T_{3}$ and $c_{1}>0$ is as Theorem 2.3. Consequently, $T_{3}$ has an infinite cluster a.s. 
Proof: The event $\left|C_{o}\left(T_{3}\right)\right|=\infty$ contains the decreasing intersection $\bigcap_{k \geq 2}\left\{Z_{k}>0\right\}$. So by Theorem 2.3, $\mathrm{P}\left[\left|C_{o}\left(T_{3}\right)\right|=\infty\right] \geq c_{1} d_{o}^{-1}$. The proof is concluded using Lemma 2.5.

We end the section with a modification of Theorem 2.3 which we shall need in Section 5 .

Definition 2.7. Let $T=(V, E, o)$ be an infinite tree rooted tree with no leaves, satisfying condition (2.2). Let $I \subset V$ be such that $d(u, v) \geq 15$, for all $u, v \in I$. Assume that $d_{v} \geq 3$, for all $v \in V \backslash I$. Let $k \geq 15$ be an odd integer. We say that a path $\gamma \in \Gamma_{k}$ is nice if it is good (i.e. in the notation of Definition 2.1, the event $A_{\gamma}=\bigcap_{0 \leq i \leq \frac{k-1}{2}} A_{i}(\gamma)$ occurs) and $\gamma \cap I \subset T_{2}$. Let $W_{o, k}$ be the union of all nice paths in $\bar{\Gamma}_{k}$.

Lemma 2.8. There exist some $b>1$ and $c_{2}>0$ (both independent of $k$ ) such that

$$
\mathrm{P}\left[\left|W_{o, k}\right|>b^{k}\right]>c_{2} / d_{0}, \text { for all odd } k \geq 15 .
$$

Proof: Let $\gamma=\left(\gamma_{0}, \ldots, \gamma_{k}\right) \in \Gamma_{k}$. Let $C_{\gamma}$ be the event that $\gamma$ is nice. For any $0 \leq i \leq \frac{k-1}{2}$ such that $I \cap\left\{\gamma_{2 i}, \gamma_{2 i+1}\right\}$ is non-empty, set $f_{i}=2 i$ and $g_{i}=2 i+1$ if $\gamma_{2 i} \in I$ and set $f_{i}=2 i+1$ and $g_{i}=2 i$ if $\gamma_{2 i+1} \in I$. For $0 \leq i \leq \frac{k-1}{2}$ let $D_{i}(\gamma)=A_{i}(\gamma)$ if $I \cap\left\{\gamma_{2 i}, \gamma_{2 i+1}\right\}$ is empty. Otherwise, set $D_{i}(\gamma)$ be the event that $\gamma_{f_{i}}$ and $\gamma_{g_{i}}$ are both younger than all their neighbors not lying in $\gamma$ (resp.) and that $\gamma_{f_{i}}$ is younger than $\gamma_{g_{i}}$. A simple calculation, similar to (2.5), shows that $4 \mathrm{P}\left[D_{i}(\gamma)\right] \geq \mathrm{P}\left[A_{\gamma}\right]$, for all $i$. Note that $D_{\gamma}:=\bigcap_{0 \leq i \leq \frac{k-1}{2}} D_{i}(\gamma) \subset C_{\gamma}$, and that $D_{0}(\gamma), D_{1}(\gamma), \ldots, D_{\frac{k-1}{2}}(\gamma)$ are jointly independent. Consequently,

$$
\mathrm{P}\left[D_{\gamma}\right] \geq \mathrm{P}\left[A_{\gamma}\right] 4^{-|A \cap \gamma|} .
$$

Similarly to $(2.1)$, set $w(\gamma):=\left[d_{o} \prod_{i=1}^{k-1}\left(d_{v_{i}}-1\right)\right]^{-1}, \bar{Y}_{\gamma}:=\frac{w(\gamma) 1_{D_{\gamma}}}{\mathrm{P}\left[D_{\gamma}\right]}$ and $\bar{Z}_{k}:=$ $\sum_{\gamma \in \Gamma_{k}} \bar{Y}_{\gamma}$.

Let $\gamma, \gamma^{\prime} \in \Gamma_{k}$ be such that $\left|\gamma \wedge \gamma^{\prime}\right| \in\{2 i, 2 i+1\}$. If $F_{\gamma, \gamma^{\prime}}$ be the event that $B_{\gamma, \gamma^{\prime}}$ occurs and that if $\left\{\gamma_{2 i}, \gamma_{2 i+1}\right\} \cap I$ or $\left\{\gamma_{2 i}^{\prime}, \gamma_{2 i+1}^{\prime}\right\} \cap I$ are non-empty, then $\gamma_{f_{i}}$ is younger than $\gamma_{g_{i}}$ or $\gamma_{f_{i}}^{\prime}$ is younger than $\gamma_{g_{i}}^{\prime}$, respectively. Then $2 \mathrm{P}\left[F_{\gamma, \gamma^{\prime}}\right] \geq \mathrm{P}\left[B_{\gamma, \gamma^{\prime}}\right]$ and similarly to (2.8), we have that

$$
\frac{\mathrm{P}\left[D_{\gamma} \cap D_{\gamma^{\prime}}\right]}{\mathrm{P}\left[D_{\gamma}\right] \mathrm{P}\left[D_{\gamma^{\prime}}\right]} \leq \frac{\mathrm{P}\left[F_{\gamma, \gamma^{\prime}}\right]}{\mathrm{P}\left[D_{i}(\gamma)\right] \mathrm{P}\left[D_{i}\left(\gamma^{\prime}\right)\right] \prod_{0 \leq r<i} \mathrm{P}\left[D_{i}(\gamma)\right]}
$$

Similarly to the proof of Theorem 2.3, we have that

$$
\mathbf{E}\left[\bar{Z}_{k}^{2}\right] \leq d_{0} / 4 c_{2} \text {, for some constant } c_{2}>0 \text {. }
$$

Hence by the Paley-Zygmund inequality (Theorem 6.2), $\mathrm{P}\left[\bar{Z}_{k}>1 / 2\right] \geq c_{2} d_{0}^{-1}$. Note that by (2.15), (2.5) and (2.6) together with the independence of $A_{0}(\gamma), \ldots$, $A_{\frac{k-1}{2}}(\gamma)$, we have $\frac{w(\gamma)}{\mathrm{P}\left[C_{\gamma}\right]} \leq b^{-k} / 2$ for some constant $b>1$ independent of $k$, for any $\gamma \in \Gamma_{k}$. Hence on the event $\bar{Z}_{k}>1 / 2$, it must be the case that $\left|W_{o, k}\right|>b^{k}$.

\section{The first 2 layers}

In this section we show that for any locally finite graph $G$ with a countable vertex set, all connected components of $T_{2}$ are a.s. finite. This demonstrates that although the marginal probabilities in $T_{2}$ would be super-critical in the independent setup, 
for any infinite connected bounded degree tree, the dependencies affect the global properties of $T_{2}$. We start with a simple lemma.

Lemma 3.1. Given $G=(V, E)$ let $\gamma=\left(v_{1}, \ldots, v_{s}\right)$ be a simple path in $G$. Suppose that $\left\{v_{1}, \ldots, v_{s}\right\} \subseteq T_{2}(G)$ and $\min \left(X_{v_{1}}, \ldots, X_{v_{s}}\right)=X_{v_{1}}$. Then the sequence $X_{v_{1}}, \ldots, X_{v_{s}}$ is monotonically increasing. Moreover, for all $2<i \in[s], v_{i} \nsim\left\{v_{j}\right.$ : $j \in[i-2]\}$. Consequently, $T_{2}(G)$ is a.s. a forest.

Proof: If the sequence was not increasing, there would be a vertex $v_{\ell} \in \gamma$ such that $X_{v_{\ell}}>X_{v_{\ell+1}}$ and $X_{v_{\ell}}>X_{v_{\ell-1}}$. But by the definition of $T_{2}$, it cannot be the case that $v_{\ell}$ belongs to $T_{2}$, a contradiction! Now, if $2<v_{i} \sim v_{j}$ for some $j \in[i-2]$, then using the monotonicity which was just established, $X_{v_{j}}<X_{v_{i-1}}<X_{v_{i}}$, so it cannot be the case that $v_{i} \in T_{2}$.

As a warm-up, we first consider the case that $G=(V, E)$ is of bounded degree, as it is significantly simpler.

Theorem 3.2. Let $G$ be a graph with a countable vertex set $V$ and $\max _{v \in V} d_{v}=$ : $\Delta<\infty$. Then, $\mathrm{P}\left[T_{2}(G)\right.$ has an infinite connected component $]=0$.

Proof: We may assume w.l.o.g. that $G$ is connected. By Lemma 3.1, the event that $v \in V$ belongs to an infinite cluster of $T_{2}$ is equal to the event that there exists some $\gamma \in \Gamma_{v, \infty}$ such that $X_{\gamma_{i}}<X_{\gamma_{i+1}}$, for all $i \in \mathbb{N}$ and that $\gamma \subset T_{2}$. Call the previous event $I_{v, \infty}$.

We now show that $\mathrm{P}\left[I_{v, \infty}\right]=0$, for all $v \in V$. Fix some $v \in V$. For every $n \in \mathbb{N}$ we define $\Gamma_{v, n}^{\prime}$ to be the collection of all $\gamma \in \Gamma_{v, n}$, such that $d_{\gamma_{\ell}} \geq 2$ and $\gamma_{i} \nsim \gamma_{j}$, for all $2 \leq \ell \leq n+1$ and all $1 \leq j \leq i-2 \leq n-1$. For every $\gamma \in \Gamma_{v, n}^{\prime}$ we define $L_{\gamma}$ to be the event that $\gamma \subset T_{2}$ and that $v$ is the youngest vertex in $\gamma$. Define $I_{v, n}:=\bigcup_{\gamma \in \Gamma_{v, n}^{\prime}} L_{\gamma}$. By Lemma 3.1

$$
\mathrm{P}\left[L_{\gamma}\right] \leq \frac{1}{(n+1) !}, \text { for all } \gamma \in \Gamma_{v, n}^{\prime}, \text { for all } n .
$$

Clearly, $\left|\Gamma_{v, n}^{\prime}\right| \leq \Delta^{n}$. Hence by (3.1) and a union bound over all $\gamma \in \Gamma_{v, n}^{\prime}$, we get that

$$
\mathrm{P}\left[I_{v, n}\right] \leq \frac{\Delta^{n}}{(n+1) !} \rightarrow 0, \text { as } n \rightarrow \infty .
$$

By Lemma 3.1, the decreasing intersection $\bigcap_{n \geq 1} I_{v, n}$ equals $I_{v, \infty}$. So $\mathrm{P}\left[I_{v, \infty}\right]=0$, for all $v \in V$. We are done, since $\mathrm{P}\left[T_{2}\right.$ has an infinite cluster $] \leq \sum_{v \in V} \mathrm{P}\left[I_{v, \infty}\right]=$ 0 .

Theorem 3.3. Let $G=(V, E)$ be a locally finite graph with a countable vertex set $V$. Then, $\mathrm{P}\left[T_{2}(G)\right.$ has an infinite connected component $]=0$.

Proof: For all $v \in V$ and $n \in \mathbb{N}$ we define $I_{v, n}$ and $\Gamma_{v, n}^{\prime}$ as in the proof of Theorem 3.2. As in the proof of Theorem 3.2, it suffices to show that $\mathrm{P}\left[I_{v, n}\right] \rightarrow 0$, as $n \rightarrow \infty$, for all $v \in V$. Instead of a straightforward union bound over all $\gamma \in \Gamma_{v, n}^{\prime}$, which is difficult to perform in the non-bounded degree setup, we perform a weighted first moment calculation which gives rise to a recurrence relation with respect to $n$.

Fix some $v \in V$ with $d_{v}>1$. For every $n>1$, we define

$\kappa(\gamma):=\prod_{i=2}^{n}\left(\frac{d_{\gamma_{i}}}{d_{\gamma_{i}}-1}\right)\left(\frac{d_{\gamma_{i}}+d_{\gamma_{i-1}}-1}{d_{\gamma_{i-1}}}\right)$, for every $\gamma:=\left(v=\gamma_{1}, \ldots, \gamma_{n+1}\right) \in \Gamma_{v, n}^{\prime}$. 
We will show that

$$
\min _{\gamma \in \Gamma_{v, n}^{\prime}} \kappa(\gamma) \rightarrow \infty, \quad \text { as } n \rightarrow \infty .
$$

For every $\gamma \in \Gamma_{v, n}^{\prime}$ let $L_{\gamma}$ be the event that $\gamma \subset T_{2}$ and that $v$ has the minimal age in $\gamma$. Let $Y_{\gamma}:=1_{L_{\gamma}}$. We will show that for all $n \in \mathbb{N}$,

$$
\mathbf{E}\left[\sum_{\gamma \in \Gamma_{v, n}^{\prime}} \kappa(\gamma) Y_{\gamma}\right] \leq M \text {, for some constant } M=M(v) \text {, independent of } n \text {. }
$$

Note that (3.3) in conjunction with (3.2) imply that $\lim _{n \rightarrow \infty} \mathbf{E}\left[\sum_{\gamma \in \Gamma_{v, n}^{\prime}} Y_{\gamma}\right]=0$. In particular, a union bound over all $\gamma \in \Gamma_{v, n}^{\prime}$ yields that

$$
\mathrm{P}\left[I_{v, n}\right]=\mathrm{P}\left[\bigcup_{\gamma \in \Gamma_{v, n}^{\prime}} L_{\gamma}\right] \leq \sum_{\gamma \in \Gamma_{v, n}^{\prime}} \mathrm{P}\left[L_{\gamma}\right]=\mathbf{E}\left[\sum_{\gamma \in \Gamma_{v, n}^{\prime}} Y_{\gamma}\right] \rightarrow 0, \text { as } n \rightarrow \infty,
$$

from which the assertion of the theorem follows.

We now prove (3.2). For all $1<m \in \mathbb{N}$ we define $n(m)$ to be the minimal integer satisfying $\left(1+\frac{1}{m d_{v}-1}\right)^{n(m)-1} \geq m$. If $\gamma \in \Gamma_{v, n(m)}^{\prime}$ and $\max \left\{d_{u}: u \in \gamma\right\} \leq m d_{v}$, then by the choice of $n(m)$, we get that $\kappa(\gamma) \geq \prod_{u \in \gamma \backslash\{v\}} \frac{d_{u}}{d_{u}-1} \geq\left(1+\frac{1}{m d_{v}-1}\right)^{n(m)-1} \geq m$. We now show that for every $\gamma \in \Gamma_{v, n(m)}^{\prime}$ with $\max \left\{d_{u}: u \in \gamma\right\}>m d_{v}$, we also have that $\kappa(\gamma) \geq m$. Fix some $\gamma \in \Gamma_{v, n(m)}^{\prime}$. Consider

$$
J:=\left\{1<i \in[n+1]: d_{\gamma_{i}}>d_{\gamma_{j}} \text { for all } j \in[i-1]\right\} .
$$

We can order the elements of $J$, as follows: $J=\left\{i_{1}, i_{2}, \ldots, i_{k}\right\}(k=|J|)$, such that if $1 \leq s<t \leq k$, then the degree of $v_{i_{s}}$ is smaller than that of $v_{i_{t}}$. For typographical reasons, for any $s \in[k]$ we denote $u_{s}:=\gamma_{i_{s}}$ and set $u_{0}=v$.

Note that if $1 \leq x \leq y \leq z$, then $\frac{x+y-1}{x} \frac{y+z-1}{y} \geq \frac{x+z-1}{x}$. Whence by induction, $\prod_{i=1}^{k} \frac{n_{i-1}+n_{i}-1}{n_{i-1}} \geq \frac{n_{0}+n_{k}-1}{n_{0}}$, for any integers $1 \leq n_{0}<n_{1}<\cdots<n_{k}$. Thus,

$$
\begin{aligned}
\kappa(\gamma) & \geq \prod_{s \in[k]} \frac{d_{\gamma_{i_{s}-1}}+d_{\gamma_{i_{s}}}-1}{d_{\gamma_{i_{s}-1}}} \geq \prod_{s \in[k]} \frac{d_{u_{s-1}}+d_{u_{s}}-1}{d_{u_{s-1}}} \\
& \geq \frac{d_{u_{0}}+d_{u_{k}}-1}{d_{u_{0}}} \geq \frac{\max \left\{d_{u}: u \in \gamma\right\}}{d_{v}} \geq m,
\end{aligned}
$$

as claimed. This establishes (3.2).

We now prove (3.3). Fix some $n \geq 2$ and some $\gamma \in \Gamma_{v, n}^{\prime}$. Let $B_{\gamma}$ be the event that $X_{\gamma_{i}}<X_{\gamma_{i+1}}$ for all $i \in[n]$ and that for all $1<i<n+1$ we have that $X_{\gamma_{i}}<X_{u}$ for all $u \notin \gamma$ such that $u \sim \gamma_{i}$. By Lemma 3.1, $B_{\gamma} \supset L_{\gamma}$. For every $1<i \leq n$, we denote

$$
T_{i}(\gamma):=\left\{u: d\left(u,\left\{\gamma_{i}, \gamma_{i+1}, \ldots, \gamma_{n}\right\}\right) \leq 1\right\} \backslash\left\{\gamma_{i-1}\right\} .
$$

Set $T_{1}(\gamma):=T_{2}(\gamma) \cup\{v\}$. For $i \in[n]$ let $C_{i}(\gamma)$ be the event that $\gamma_{i}$ is the youngest vertex in $T_{i}(\gamma)$. Note that $B_{\gamma}=\bigcap_{i=1}^{n} C_{i}(\gamma)$. Observe that the events $C_{1}(\gamma), \ldots, C_{n}(\gamma)$ are mutually independent. One way to see this is to note that the conditional distribution of $\left(X_{u}: u \in T_{i+1}(\gamma)\right)$, given $C_{1}(\gamma), \ldots, C_{i}(\gamma)$ and $\left(X_{\gamma_{j}}: j \in[i]\right)$, is that of independent $\operatorname{Uniform}\left(X_{\gamma_{i}}, 1\right]$ random variables. Alternatively, this follows from the fact that all orderings of $T_{1}(\gamma)$ (with respect to the ages 
of the vertices of $\left.T_{1}(\gamma)\right)$ are equally likely. Thus,

$$
\mathrm{P}\left[B_{\gamma}\right]=\prod_{i=1}^{n} \mathrm{P}\left[C_{i}(\gamma)\right]=\prod_{i=1}^{n} \frac{1}{\left|T_{i}(\gamma)\right|} .
$$

Let $m(\gamma):=\left\{u \sim \gamma_{n+1}: d\left(u,\left\{\gamma_{j}: j \in[n]\right\}\right) \geq 2\right\}$. For every $\gamma^{\prime}:=\left(\gamma_{1}^{\prime}, \gamma_{2}^{\prime}, \ldots, \gamma_{n+2}^{\prime}\right)$ $\in \Gamma_{v, n+1}^{\prime}$ we denote $\left.\gamma^{\prime}\right|_{[n+1]}:=\left(\gamma_{1}^{\prime}, \ldots, \gamma_{n+1}^{\prime}\right)$. From the definition of $\Gamma_{v, n+1}^{\prime}$, if $\gamma^{\prime} \in \Gamma_{v, n+1}$ is such that $\left.\gamma^{\prime}\right|_{[n+1]}=\gamma$, then $\gamma_{n+2}^{\prime} \in m(\gamma)$. Moreover, the following hold.

$$
\begin{gathered}
\kappa\left(\gamma^{\prime}\right)=\kappa(\gamma) \frac{d_{\gamma_{n+1}}\left(d_{\gamma_{n}}+d_{\gamma_{n+1}}-1\right)}{\left(d_{\gamma_{n+1}}-1\right) d_{\gamma_{n}}} \\
\left|T_{i}(\gamma)\right| \leq\left|T_{i}\left(\gamma^{\prime}\right)\right|, \text { for all } i \in[n-1] \\
\left|T_{n+1}\left(\gamma^{\prime}\right)\right|=d_{\gamma_{n+1}},\left|T_{n}(\gamma)\right|=d_{\gamma_{n}} \text { and }\left|T_{n}\left(\gamma^{\prime}\right)\right| \geq d_{\gamma_{n}}+|m(\gamma)| \\
|m(\gamma)| \leq d_{\gamma_{n+1}}-1 .
\end{gathered}
$$

Hence, by (3.4) we have

$$
\begin{gathered}
\sum_{\gamma^{\prime} \in \Gamma_{v, n+1}^{\prime}:\left.\gamma^{\prime}\right|_{[n+1]}=\gamma} \mathrm{P}\left[B_{\gamma^{\prime}}\right] \kappa\left(\gamma^{\prime}\right)=\sum_{\gamma^{\prime} \in \Gamma_{v, n+1}^{\prime}:\left.\gamma^{\prime}\right|_{[n+1]}=\gamma} \kappa\left(\gamma^{\prime}\right) \prod_{i=1}^{n+1} 1 /\left|T_{i}\left(\gamma^{\prime}\right)\right| \\
\leq \kappa(\gamma) \frac{d_{\gamma_{n+1}}\left(d_{\gamma_{n}}+d_{\gamma_{n+1}}-1\right)}{\left(d_{\gamma_{n+1}}-1\right) d_{\gamma_{n}}} \prod_{i=1}^{n}\left|T_{i}(\gamma)\right|^{-1} \sum_{\gamma^{\prime} \in \Gamma_{v, n+1}^{\prime}:\left.\gamma^{\prime}\right|_{[n+1]}=\gamma} \frac{\left|T_{n}(\gamma)\right|}{d_{\gamma_{n+1}}\left|T_{n}\left(\gamma^{\prime}\right)\right|} \\
\leq \kappa(\gamma) \frac{d_{\gamma_{n+1}}\left(d_{\gamma_{n}}+d_{\gamma_{n+1}}-1\right)}{\left(d_{\gamma_{n+1}}-1\right) d_{\gamma_{n}}} \mathrm{P}\left[B_{\gamma}\right]|m(\gamma)| \frac{d_{\gamma_{n}}}{d_{\gamma_{n+1}}\left(d_{\gamma_{n}}+|m(\gamma)|\right)} \leq \mathrm{P}\left[B_{\gamma}\right] \kappa(\gamma) .
\end{gathered}
$$

Denote $Z_{\gamma}:=1_{B_{\gamma}}$. From (3.5) we get the following recurrence relation,

$$
\begin{aligned}
\mathbf{E}\left[\sum_{\gamma^{\prime} \in \Gamma_{v, n+1}^{\prime}} \kappa\left(\gamma^{\prime}\right) Z_{\gamma^{\prime}}\right] & \leq \mathbf{E}\left[\sum_{\gamma \in \Gamma_{v, n}^{\prime} \gamma^{\prime} \in \Gamma_{v, n+1}^{\prime}:\left.\gamma^{\prime}\right|_{[n+1]}=\gamma} \kappa\left(\gamma^{\prime}\right) Z_{\gamma^{\prime}}\right] \\
& \leq \mathbf{E}\left[\sum_{\gamma \in \Gamma_{v, n}^{\prime}} \kappa(\gamma) Z_{\gamma}\right] .
\end{aligned}
$$

Iterating, we get that $\mathbf{E}\left[\sum_{\gamma^{\prime} \in \Gamma_{v, n+1}^{\prime}} \kappa\left(\gamma^{\prime}\right) Z_{\gamma^{\prime}}\right] \leq \mathbf{E}\left[\sum_{\gamma^{\prime} \in \Gamma_{v, 2}^{\prime}} \kappa\left(\gamma^{\prime}\right) Z_{\gamma^{\prime}}\right]=: M(v)$, for every $1<n \in \mathbb{N}$. This implies (3.3), since $Y_{\gamma^{\prime}} \leq Z_{\gamma^{\prime}}$ for all $\gamma^{\prime} \in \Gamma_{v, n+1}$ (as $\left.B_{\gamma^{\prime}} \supset L_{\gamma^{\prime}}\right)$.

\section{The first 4 layers in $\mathbb{Z}^{d}$}

For every $1 \leq i \leq d$, let $e_{i} \in \mathbb{Z}^{d}$ be the vector whose $i$ th co-ordinate is 1 and the rest of its co-ordinates are 0 . Let

$$
\mathbb{Z}_{+}^{d}:=\left\{\left(x_{1}, \ldots, x_{d}\right) \in \mathbb{Z}^{d}: x_{i} \geq 0, i \in[d]\right\} .
$$

In this section we prove the following theorem, whose assertion is stronger than that of Theorem 1.4.

Theorem 4.1. For all sufficiently large $d$, the vertex-induced graph on $T_{4}\left(\mathbb{Z}^{d}\right) \cap \mathbb{Z}_{+}^{d}$ a.s. contains an infinite path $\left(v_{1}, v_{2}, \ldots\right)$, such that $v_{i+1}-v_{i} \in\left\{e_{j}: j \in[d]\right\}$, for all $i$. 
In the proof of Theorem 4.1 we use a variant of the EIT method, introduced in Cox and Durrett (1983). Cox and Durrett attribute the argument to Harry Kesten. As a warm-up, we first present in Lemma 4.4, a calculation taken from Cox and Durrett (1983). We do not use Lemma 4.4 and we present it and its proof since the proof of Lemma 4.5 uses some of the calculations from the proof of Lemma 4.4.

Definition 4.2 (EIT). Let $\mu$ be a probability measure on infinite simple paths in a graph $G$. Let $\alpha \in(0,1)$. We say that $\mu$ has $\operatorname{EIT}(\alpha)$, if there exists some $C>0$ such that for all $k \in \mathbb{N}$,

$$
\mu \times \mu\left(\left\{\left(\gamma, \gamma^{\prime}\right):\left|\gamma \cap \gamma^{\prime}\right| \geq k\right\}\right) \leq C \alpha^{k},
$$

where $\left|\gamma \cap \gamma^{\prime}\right|$ is the number of vertices the paths $\gamma$ and $\gamma^{\prime}$ have in common. In simple words, the probability that two paths chosen independently, each from the distribution $\mu$, will have at least $k$ common vertices, is at most $C \alpha^{k}$. If such $\mu$ exists, we say that $G$ admits random paths with $\operatorname{EIT}(\alpha)$. The same definition applies when $G$ is an oriented graph and the paths are oriented paths.

Definition 4.3. Consider a random walk with initial position $0:=(0,0, \ldots, 0)$, whose increments distribution is the uniform distribution on $\left\{e_{i}: i \in[d]\right\}$. Let $\mu_{d}$ be the probability measure corresponding to the infinite trajectory of this random walk. Then $\mu_{d}$ is called the uniform distribution on monotone paths in $\mathbb{Z}^{d}$. We call a path $\gamma$ a monotone path if $\gamma_{i+1}-\gamma_{i} \in\left\{e_{j}: j \in[d]\right\}$, for all $i \in \mathbb{N}$. We denote the collection of all monotone paths of length $k$ starting from $\mathbf{0}$ by $\Gamma_{k}^{\text {mon }}$.

Lemma 4.4. $\mu_{d}$ has $\operatorname{EIT}\left(\alpha_{d}\right)$ for some $\alpha_{d}=1 / d+(1 / d)^{2}+O\left(d^{-3}\right)$, for any $d \geq 4$.

Proof: Let $\left(S_{k}\right)_{k=0}^{\infty}$ and $\left(S_{k}^{\prime}\right)_{k=0}^{\infty}$ be two independent random walks with distribution $\mu_{d}$. Let $\tau:=\inf \left\{k \geq 1: S_{k}=S_{k}^{\prime}\right\}$. Then,

$$
\begin{aligned}
& \mathrm{P}(\tau=1)=d^{-1}, \\
& \mathrm{P}(\tau=2)=d^{-2}-d^{-3}, \\
& \mathrm{P}(\tau=3)<3 d^{-3},
\end{aligned}
$$

as

$$
\begin{aligned}
& \mathrm{P}(\tau=3)= \\
& \mathrm{P}\left[d\left(S_{1}, S_{1}^{\prime}\right)=2\right] \mathrm{P}\left[d\left(S_{2}, S_{2}^{\prime}\right)=2 \mid d\left(S_{1}, S_{1}^{\prime}\right)=2\right] \mathrm{P}\left[d\left(S_{3}, S_{3}^{\prime}\right)=0 \mid d\left(S_{2}, S_{2}^{\prime}\right)=2\right] \\
& <3 d^{-3} .
\end{aligned}
$$

By the independence of $\left(S_{k}\right)_{k=0}^{\infty}$ and $\left(S_{k}^{\prime}\right)_{k=0}^{\infty}$,

$$
\begin{aligned}
\mathrm{P}(\tau=k) \leq \mathrm{P}\left(S_{k}=S_{k}^{\prime}\right) & =\sum_{x} \mathrm{P}\left(S_{k}=x\right) \mathrm{P}\left(S_{k}^{\prime}=x\right) \\
& \leq \max _{x} \mathrm{P}\left(S_{k}=x\right), \text { for any } k \geq 4 .
\end{aligned}
$$

If $4 \leq k \leq d$, then for any $x \in \mathbb{Z}_{+}^{d}$, with $d(x, 0)=k$, we have

$$
\mathrm{P}\left(S_{k}=x\right)=\left(\begin{array}{c}
k \\
x_{1}, \ldots, x_{d}
\end{array}\right) d^{-k} \leq k ! d^{-k} .
$$

Since the right hand side of (4.3) is non-increasing in $k$ for $4 \leq k \leq d,(4.2)-(4.3)$ imply that,

$$
\mathrm{P}(4 \leq \tau \leq d) \leq \sum_{k=4}^{d} k ! d^{-k} \leq 4 ! d^{-4}+5 ! d^{-5}+(d-6) 6 ! d^{-6}=O\left(d^{-4}\right) .
$$


If $d \ell \leq k<d(\ell+1)$ for some $\ell \in \mathbb{N}$, then for any $x \in \mathbb{Z}_{+}^{d}$ with $d(x, 0)=k$,

$$
\begin{aligned}
P\left(S_{k}=x\right) & =\left(\begin{array}{c}
k \\
x_{1}, \ldots, x_{d}
\end{array}\right) d^{-k} \leq \frac{k !}{\ell !^{d-k+d \ell}(\ell+1) ! k-d \ell} d^{-k} \\
& =\frac{(d \ell) !}{\ell !^{d} d^{d \ell}} \prod_{i=1}^{k-d \ell} \frac{d \ell+i}{d(\ell+1)} \leq \frac{(d \ell) !}{\ell !^{d} d^{d \ell}} .
\end{aligned}
$$

By Stirling's formula (e.g. Feller, 1971 page 54),

$$
e^{-1 / 13} \leq \frac{n !}{n^{n} e^{-n} \sqrt{2 \pi n}} \leq 1, \text { for all } n \in \mathbb{N}
$$

Plugging this estimate in (4.5), we get by (4.2) that:

$$
\begin{aligned}
\mathrm{P}(\tau>d) & \leq \sum_{\ell=1}^{\infty} \mathrm{P}(d \ell \leq \tau<d(\ell+1)) \leq \sum_{\ell=1}^{\infty} d \frac{(d \ell) !}{\ell ! d d^{d \ell}} \\
& \leq \sum_{\ell=1}^{\infty} \frac{(d l)^{d \ell} d(2 \pi d \ell)^{1 / 2}}{\left(\ell^{\ell} e^{-\frac{1}{13}} \sqrt{2 \pi \ell}\right)^{d} d^{d \ell}}=d^{3 / 2} \sqrt{2 \pi}\left(\frac{e^{\frac{1}{13}}}{\sqrt{2 \pi}}\right)^{d} \sum_{\ell=1}^{\infty} \ell^{(1-d) / 2} .
\end{aligned}
$$

The last sum is finite since $d \geq 4$. Since $e^{1 / 13}<\sqrt{2 \pi}$, the last expression approaches zero exponentially rapidly as $d \rightarrow \infty$.

In conclusion, $\mathrm{P}(\tau<\infty) \leq 1 / d+(1 / d)^{2}+O\left(d^{-3}\right)$. The assertion of the lemma now follows from the strong Markov property, applied to the random walk $\left(S_{k}-\right.$ $\left.S_{k}^{\prime}\right)_{k=0}^{\infty}$.

We adopt the convention that for any $i \in \mathbb{N}, O\left(d^{-i}\right)$ can be a negative term whose absolute value is $O\left(d^{-i}\right)$ in the usual sense.

Lemma 4.5. Let $\left(S_{k}\right)_{k=0}^{\infty}$ and $\left(S_{k}^{\prime}\right)_{k=0}^{\infty}$ be two independent random walks with distribution $\mu_{d}$. Denote

$$
\begin{aligned}
p_{1,2,3,4} & :=\mathrm{P}\left[\left\{\exists n>2, d\left(S_{n}, S_{n}^{\prime}\right)=2\right\} \mid S_{2}=e_{1}+e_{3}, S_{2}^{\prime}=e_{2}+e_{4}\right], \\
p_{1,2} & :=\mathrm{P}\left[\left\{\exists n>2, d\left(S_{n}, S_{n}^{\prime}\right)=2\right\} \mid S_{2}=2 e_{1}, S_{2}^{\prime}=2 e_{2}\right], \\
p_{1,2,3} & :=\mathrm{P}\left[\left\{\exists n>2, d\left(S_{n}, S_{n}^{\prime}\right)=2\right\} \mid S_{2}=2 e_{1}, S_{2}^{\prime}:=e_{2}+e_{3}\right], \\
a_{2} & :=\mathrm{P}\left[\left\{\exists n>1, d\left(S_{n}, S_{n}^{\prime}\right)=0\right\} \mid S_{1}=e_{1}, S_{1}^{\prime}=e_{2}\right] .
\end{aligned}
$$

Then,

$$
\begin{aligned}
a_{2} & =d^{-2}+O\left(d^{-3}\right), \\
p_{1,2} & =d^{-2}+O\left(d^{-3}\right), \\
p_{1,2,3} & =2 d^{-2}+O\left(d^{-3}\right), \\
p_{1,2,3,4} & =4 d^{-2}+O\left(d^{-3}\right) .
\end{aligned}
$$

Proof: Let $\tau:=\inf \left\{k \geq 1: S_{k}=S_{k}^{\prime}\right\}$. By symmetry of the lattice

$$
a_{2}=\mathrm{P}[\tau<\infty \mid \tau>1]=\frac{\mathrm{P}[1<\tau<\infty]}{\mathrm{P}[\tau>1]}=\frac{d^{-2}+O\left(d^{-3}\right)}{1-d^{-1}}=d^{-2}+O\left(d^{-3}\right) .
$$

Let $\tau^{\prime}:=\tau-4$. Note that if $d\left(S_{2}, S_{2}^{\prime}\right)=4$, then $\tau^{\prime} \geq 0$. Moreover, $\mathrm{P}\left[\tau^{\prime}=0 \mid\right.$ $\left.\left(S_{2}, S_{2}^{\prime}\right)=\left(2 e_{1}, 2 e_{2}\right)\right]=d^{-4}, \mathrm{P}\left[\tau^{\prime}=0 \mid\left(S_{2}, S_{2}^{\prime}\right)=\left(2 e_{1}, e_{2}+e_{3}\right)\right]=2 d^{-4}$ and 
$\mathrm{P}\left[\tau^{\prime}=0 \mid\left(S_{2}, S_{2}^{\prime}\right)=\left(e_{1}+e_{3}, e_{2}+e_{4}\right)\right]=4 d^{-4}$. Similarly to the proof of Lemma 4.4 , for any $\left(z, z^{\prime}\right) \in\left\{\left(2 e_{1}, 2 e_{2}\right),\left(2 e_{1}, e_{2}+e_{3}\right),\left(e_{1}+e_{3}, e_{2}+e_{4}\right)\right\}$ we have that

$$
\begin{aligned}
& \mathrm{P}\left[1 \leq \tau^{\prime}<\infty \mid\left(S_{2}, S_{2}^{\prime}\right)=\left(z, z^{\prime}\right)\right] \leq \sum_{k=3}^{\infty} \mathrm{P}\left[S_{k+2}-S_{k+2}^{\prime}=\mathbf{0} \mid\left(S_{2}, S_{2}^{\prime}\right)=\left(z, z^{\prime}\right)\right]= \\
& \sum_{k=3}^{\infty} \mathrm{P}\left[S_{k}-S_{k}^{\prime}=z^{\prime}-z\right] \leq 3 !^{2} d^{-5}+4 !^{2} d^{-6}+\sum_{k=5}^{\infty} \max _{x} \mathrm{P}\left(S_{k}=x\right)=O\left(d^{-5}\right) .
\end{aligned}
$$

By the strong Markov property,

$$
p_{1,2}:=\frac{\mathrm{P}\left[\tau^{\prime}<\infty \mid\left(S_{2}, S_{2}^{\prime}\right)=\left(2 e_{1}, 2 e_{2}\right)\right]}{a_{2}}=\frac{d^{-4}+O\left(d^{-5}\right)}{d^{-2}+O\left(d^{-3}\right)}=d^{-2}+O\left(d^{-3}\right) .
$$

The proofs of the last two equations in (4.7) are concluded in the same manner.

Proof of Theorem 4.1: Let $\gamma \in \Gamma_{2 k-1}^{\mathrm{mon}}$ (where $\Gamma_{2 k-1}^{\mathrm{mon}}$ is as in Definition 4.3). To have symmetry in our construction, for reasons that shall soon be clear, we define $\gamma_{0}:=-e_{1}$ and $\gamma_{2 k+1}:=\gamma_{2 k}+e_{1}$. For any $i \in[k]$, we define

$$
M_{2 i-1}(\gamma):=\left|\left\{u \sim \gamma_{2 i-1}: u \neq \gamma_{2 i-2}, X_{u}<X_{\gamma_{2 i-1}}\right\}\right| \text {, }
$$

and

Define

$$
M_{2 i}(\gamma):=\left|\left\{u \sim \gamma_{2 i}: u \neq \gamma_{2 i+1}, X_{u}<X_{\gamma_{2 i}}\right\}\right|
$$

and denote

$$
A_{i}(\gamma):=\left\{M_{2 i-1}(\gamma) \leq 2\right\} \cap\left\{M_{2 i}(\gamma) \leq 2\right\}
$$

$$
A(\gamma):=\bigcap_{i=1}^{k} A_{i}(\gamma)
$$

By construction, on $A(\gamma), \gamma$ is contained in $T_{4}\left(\mathbb{Z}^{d}\right)$. Notice that $\gamma_{2 i-1}$ and $\gamma_{2 i}$ do not have a common neighbor, for all $i \in[k]$. Whence, similarly to the proof of Theorem 2.3, we can calculate $\mathrm{P}\left[A_{i}(\gamma)\right]$ by a direct calculation which is completely elementary.

Proposition 4.6. For all $i \in[k]$ we have that $\mathrm{P}\left[A_{i}(\gamma)\right] \geq(a / d)^{2}$ for some absolute constant $a>1$.

Proof of Proposition 4.6: For the sake of concreteness, we show that for $i \in[k]$,

$$
\begin{aligned}
\mathrm{P}\left[A_{i}(\gamma)\right] & =\left(\frac{2}{2 d-1}\right)^{2}+\frac{(2 d-3)}{(4 d-2)(4 d-3)(2 d-1)}+\frac{3(2 d-3)}{(4 d-2)(4 d-3)(4 d-5)} \\
& >\frac{9}{8 d^{2}} .
\end{aligned}
$$

Before explaining this inequality we note that the exact term $9 / 8$ shall not be used in what comes. Denote

$$
M_{j}^{\prime}(\gamma):=\left|\left\{u \sim \gamma_{j}: u \notin \gamma, X_{u}<X_{\gamma_{j}}\right\}\right|, j=2 i-1,2 i .
$$

The first term above comes from the case that $M_{2 i-1}^{\prime}(\gamma), M_{2 i}^{\prime}(\gamma) \leq 1$. The middle term comes from the case that $M_{2 i}^{\prime}(\gamma)=2, M_{2 i-1}^{\prime}(\gamma) \leq 1$ and $\gamma_{2 i}$ is younger than all of the vertices in $\left\{u: u \sim \gamma_{2 i-1}, u \notin \gamma\right\} \cup\left\{\gamma_{2 i-1}\right\}$ and the corresponding case in which the roles of $2 i$ and $2 i-1$ are reversed. The last term comes from the case that $M_{2 i}^{\prime}(\gamma)=2, M_{2 i-1}^{\prime}(\gamma)=1$ and the unique neighbor of $\gamma_{2 i-1}$ not belonging to $\gamma$ which is younger than $\gamma_{2 i-1}$ is also younger than $\gamma_{2 i}$ which in turn is younger 
than $\gamma_{2 i-1}$, together with the corresponding case where the roles of $2 i$ and $2 i-1$ are reversed.

Note that the events $A_{1}(\gamma), \ldots, A_{k}(\gamma)$ are usually not independent, since vertices of distance two in $\gamma$ can have a common neighbor. Hence we need the following proposition. Recall that in our convention $v \sim A \subset V$ iff $d(v, A)=1$ (in particular $v \notin A)$.

Proposition 4.7. Let $\gamma \in \Gamma_{2 k-1}^{\text {mon }}$. Denote $X:=\left(X_{a}: a \sim \gamma\right), Y:=\left(X_{u}: u \in \gamma\right)$.

(i) The events $A_{1}(\gamma), \ldots, A_{k}(\gamma)$ are positively correlated.

(ii) The conditional distribution of $X$, given $A(\gamma)$, stochastically dominates its unconditional distribution.

Proof of Proposition 4.\%: Fix some $\gamma:=\left(v_{1}, \ldots, v_{2 k}\right) \in \Gamma_{2 k-1}^{\text {mon }}$. Let $[0,1]^{\gamma}$ (resp. $[0,1]^{\{a: a \sim \gamma\}}$ ) be the collection of vectors whose co-ordinates take values in $[0,1]$ and are labeled by the set $\gamma$ (resp. $\{a: a \sim \gamma\}$ ). For any $j \in[k]$, let $f_{j}(X, Y)$ be the indicator of $A_{j}(\gamma)$. Observe that $f_{1}\left(X^{\prime}, Y^{\prime}\right), \ldots, f_{k}\left(X^{\prime}, Y^{\prime}\right)$ are increasing functions of $X^{\prime}\left(X^{\prime} \in[0,1]^{\{a: a \sim \gamma\}}\right)$, for any fixed $Y^{\prime} \in[0,1]^{\gamma}$.

For any $w \in\{1,2\}^{k}$, we define a partial order $\prec_{w}$ on $\gamma$ as follows. For any $j \in[k]$, if $w(j)=1$, then $v_{2 j} \prec_{w} v_{2 j-1}$ and if $w(j)=2$, then $v_{2 j-1} \prec_{w} v_{2 j}$ (and these are the only relations in $\left.\prec_{w}\right)$. Let $S_{w}$ be the event that for all $i \in[k], X_{v_{2 i-1}}<X_{v_{2 i}}$ iff $v_{2 i-1} \prec_{w} v_{2 i}$. For any $w \in\{1,2\}^{k}$ let $Y_{w}$ be a random vector distributed as $Y$ conditioned on $S_{w}$. Denote by $\mathbf{E}_{X}$ the expectation with respect to $X$ where $Y$ (or $\left.Y_{w}\right)$ is treated as a constant vector. For each $w \in\{1,2\}^{k}$, we say that $Z \in[0,1]^{\gamma}$ respects $\prec_{w}$ if $Z_{v_{2 i-1}}<Z_{v_{2 i}}$ iff $v_{2 i-1} \prec_{w} v_{2 i}$.

Let $Z_{1}, Z_{2} \in[0,1]^{\gamma}$ and $w \in\{1,2\}^{k}$. Note that if $Z_{1} \geq Z_{2}$ coordinate-wise and both vectors respect $\prec_{w}$, then for any $j \in[k], f_{j}\left(X^{\prime}, Z_{2}\right) \geq f_{j}\left(X^{\prime}, Z_{1}\right)$, for all $X^{\prime} \in$ $[0,1]^{\{a: a \sim \gamma\}}$. Consequently, $\mathbf{E}_{X}\left[f_{j}\left(X, Y_{w}\right)\right]$ (a shorthand for $\mathbf{E}_{X}\left[f_{j}(X, Y) \mid S_{w}\right]$ ) is a decreasing function of $Y_{w}$, for any $j \in[k]$ and $w \in\{1,2\}^{k}$. Let $\mathbf{E}_{Y_{w}}$ be the expectation with respect to $Y$ conditioned on $S_{w}$ (that is, $\mathbf{E}_{Y_{w}}[\cdot]=\mathbf{E}_{Y}\left[\cdot \mid S_{w}\right]$ ). Observe that by symmetry we have that

$$
\mathrm{P}\left[A_{i}(\gamma) \mid S_{w}\right]=\mathrm{P}\left[A_{i}(\gamma)\right] \text {, for any } i \in[k] \text { and } w \in\{1,2\}^{k} \text {. }
$$

Let $G(X)$ be the indicator function of some increasing event $B$ with respect to $X$. By an application of the FKG inequality (first and second inequalities) and of the correlation inequality for affiliated random variables from Theorem 6.4 (third 
inequality) we have that,

$$
\begin{aligned}
& \mathrm{P}\left[B \cap A(\gamma) \cap S_{w}\right]=\mathrm{P}\left[S_{w}\right] \mathbf{E}_{Y_{w}, X}\left[g(X) \prod_{j \in[k]} f_{j}\left(X, Y_{w}\right)\right] \\
& =\mathrm{P}\left[S_{w}\right] \mathbf{E}_{Y_{w}} \mathbf{E}_{X}\left[g(X) \prod_{j \in[k]} f_{j}\left(X, Y_{w}\right)\right] \geq \mathrm{P}\left[S_{w}\right] \mathbf{E}[g(X)] \mathbf{E}_{Y_{w}} \mathbf{E}_{X}\left[\prod_{j \in[k]} f_{j}\left(X, Y_{w}\right)\right] \\
& \geq \mathbf{E}[g(X)] \mathrm{P}\left[S_{w}\right] \mathbf{E}_{Y_{w}}\left[\prod_{j \in[k]} \mathbf{E}_{X}\left[f_{j}\left(X, Y_{w}\right)\right]\right] \geq \mathrm{P}[B] \mathrm{P}\left[S_{w}\right] \prod_{j \in[k]} \mathbf{E}_{Y_{w}} \mathbf{E}_{X}\left[f_{j}\left(X, Y_{w}\right)\right] \\
& =\mathrm{P}[B] \mathrm{P}\left[S_{w}\right] \prod_{j \in[k]} \mathrm{P}\left[A_{j}(\gamma) \mid S_{w}\right]=\mathrm{P}[B] \mathrm{P}\left[S_{w}\right] \prod_{j \in[k]} \mathrm{P}\left[A_{j}(\gamma)\right] .
\end{aligned}
$$

Taking $B$ to equal the entire probability space and summing over all $w \in\{1,2\}^{k}$ give that

$$
\mathrm{P}[A(\gamma)]=\mathrm{P}\left[\bigcap_{j \in[k]} A_{j}(\gamma)\right] \geq \prod_{j \in[k]} \mathrm{P}\left[A_{j}(\gamma)\right]
$$

Similarly, for any disjoint $I_{1}, I_{2} \subset[k]$, by repeating the calculations in (4.8) and the reasoning leading to $(4.9)$, one can show that if we denote $J_{i}:=\bigcap_{j \in I_{i}} A_{j}(\gamma)$ $(i=1,2)$, then

$$
\mathrm{P}\left[J_{1} \cap J_{2}\right] \geq \mathrm{P}\left[J_{1}\right] \mathrm{P}\left[J_{2}\right] .
$$

Moreover, by the first inequality in (4.8),

$\mathrm{P}\left[B \cap A(\gamma) \cap S_{w}\right] \geq \mathrm{P}\left[S_{w}\right] \mathbf{E}[g(X)] \mathbf{E}_{Y_{w}} \mathbf{E}_{X}\left[\prod_{j \in[k]} f_{j}\left(X, Y_{w}\right)\right]=\mathrm{P}[B] \mathrm{P}\left[A(\gamma) \cap S_{w}\right]$, for any $w \in\{1,2\}^{[k]}$. Summing over all $w$ we get that $\mathrm{P}[B \mid A(\gamma)] \geq \mathrm{P}[B]$.

Set

$$
Y_{\gamma}:=\frac{1_{A(\bar{\gamma})}}{\mathrm{P}[A(\bar{\gamma})]} \text { for all } \gamma \in \Gamma_{2 k-1}^{\mathrm{mon}} \text { and } Z_{k}:=\left|\Gamma_{2 k-1}^{\mathrm{mon}}\right|^{-1} \sum_{\bar{\gamma} \in \Gamma_{2 k-1}} Y_{\bar{\gamma}}
$$

Clearly $\mathbf{E}\left[Z_{k}\right]=1$. Similarly to the proof of Theorem 2.3, in order to prove the assertion of Theorem 4.1 it suffices to show that for some positive constant $\beta$ (which may depend only on $d$ ) we have that

$$
\mathbf{E}\left[Z_{k}^{2}\right] \leq \beta,
$$

since then, by Cauchy-Schwarz inequality (or Theorem 6.2 for $r=0$ )

$$
\mathrm{P}\left[Z_{k}>0\right] \geq \frac{1}{\mathbf{E}\left[Z_{k}^{2}\right]} \geq \beta^{-1}>0 .
$$

The event that $T_{4}\left(\mathbb{Z}^{d}\right) \cap \mathbb{Z}_{+}^{d}$ contains an infinite monotone path is a tail event. Since it contains the decreasing intersection of the events $\left(\left\{Z_{k}>0\right\}: k \in \mathbb{N}\right),(4.13)$ and the $0-1$ law of Lemma 2.5 imply the assertion of the theorem. 
Let $\gamma=\left(v_{1}, \ldots, v_{2 k}\right), \gamma^{\prime}=\left(v_{1}^{\prime}, \ldots, v_{2 k}^{\prime}\right) \in \Gamma_{2 k-1}^{\text {mon }}$. As in the proof of Theorem 2.3, in order to estimate $\mathbf{E}\left[Z_{k}^{2}\right]$ from above, we need to estimate $\mathrm{P}[A(\gamma) \cap$ $\left.A\left(\gamma^{\prime}\right)\right] /\left(\mathrm{P}[A(\gamma)] \mathrm{P}\left[A\left(\gamma^{\prime}\right)\right]\right)$ from above.

As before, set $v_{0}, v_{0}^{\prime}:=-e_{1}$ and $v_{2 k+1}^{\prime}:=v_{2 k}^{\prime}+e_{1}, v_{2 k+1}:=v_{2 k}+e_{1}$. Observe that if $i \in[2 k]$ and $d\left(v_{i}, v_{i}^{\prime}\right)=2$, then given $A(\gamma)$, the conditional distribution of $X_{v_{i}^{\prime}}$ is different than its unconditional distribution only if $d\left(v_{i}^{\prime},\left\{v_{i-1}, v_{i+1}\right\}\right)=1$, in which case by Proposition 4.7 (ii), its conditional distribution stochastically dominates its unconditional distribution. This can only decrease the probability of the event $A_{\lceil i / 2\rceil}\left(\gamma^{\prime}\right)$ (hence plays in our favor).

For any $i \in[2 k]$, set

$$
N_{i}\left(\gamma, \gamma^{\prime}\right)=N_{i}:=\left\{u \sim v_{i}^{\prime}: u \notin \gamma^{\prime} \cup\left\{v_{2 k+1}^{\prime}\right\}, d(u, \gamma) \leq 1\right\} .
$$

If $d\left(v_{i}, v_{i}^{\prime}\right)=2$, then $\left|N_{i}\right| \leq 4$. Note that if $d\left(v_{i}, v_{i}^{\prime}\right)=2$, then the distribution of $X_{v_{i}^{\prime}}$ and of $\left(X_{u}: u \sim v_{i}^{\prime}, u \notin N_{i} \cup \gamma\right)$ is unaffected by the occurrence of the event $A(\gamma)$. Similarly to the proof of Theorem 2.3 where we considered the event $B_{\gamma, \gamma^{\prime}}$, we now define an event which contains $A\left(\gamma^{\prime}\right)$, whose conditional distribution, given $A(\gamma)$, is easier to estimate than that of $A\left(\gamma^{\prime}\right)$. We define $M_{j}\left(\gamma^{\prime}\right)$ in an analogous manner to the definition of $M_{j}(\gamma)$. Namely, $M_{2 i-1}\left(\gamma^{\prime}\right):=\mid\left\{u \sim v_{2 i-1}^{\prime}: u \neq v_{2 i-2}^{\prime}, X_{u}<\right.$ $\left.X_{v_{2 i-1}^{\prime}}\right\} \mid$ and $M_{2 i}\left(\gamma^{\prime}\right):=\left|\left\{u \sim v_{2 i}^{\prime}: u \neq v_{2 i+1}^{\prime}, X_{u}<X_{v_{2 i}^{\prime}}\right\}\right|$. For any $j \in[2 k]$, we define

$$
K_{j}:= \begin{cases}M_{j}(\gamma), & d\left(v_{j}, v_{j}^{\prime}\right)=0, \\ \left|\left\{u \sim v_{j}^{\prime}: u \notin \gamma \cup N_{j}, u \nsim \gamma^{\prime} \backslash\left\{v_{j}^{\prime}\right\}, X_{u}<X_{v_{j}^{\prime}}\right\}\right|, & d\left(v_{j}, v_{j}^{\prime}\right)=2, \\ M_{j}\left(\gamma^{\prime}\right), & d\left(v_{j}, v_{j}^{\prime}\right)>2 .\end{cases}
$$

Define $C_{j}=C_{j}\left(\gamma, \gamma^{\prime}\right):=\left\{K_{j} \leq 2\right\}$ and $C=C\left(\gamma, \gamma^{\prime}\right):=\bigcap_{i \in[2 k]} C_{j}$. Note that $A(\gamma) \cap C \supset A(\gamma) \cap A\left(\gamma^{\prime}\right)$. Using the above observations, we get the following inequality.

Proposition 4.8. Assume $d>4$. Let a be as in Proposition 4.6. Let $j_{0}, j_{2} \in \mathbb{N}$ be such that $j_{0}+j_{2} \leq 2 k$. Assume that $v_{i}=v_{i}^{\prime}$, for any $1 \leq i \leq j_{0}$, that $d\left(v_{i}, v_{i}^{\prime}\right)=2$, for any $j_{0}+1 \leq i \leq j_{0}+j_{2}$, and that $d\left(v_{i}, v_{i}^{\prime}\right) \geq 4$, for any $j_{0}+j_{2}+1 \leq i \leq 2 k$. Then there exists an absolute constant $a^{\prime}$ such that $1<a^{\prime} \leq a$ and

$$
\mathrm{P}\left[A(\gamma) \cap C\left(\gamma, \gamma^{\prime}\right)\right] \leq \mathrm{P}[A(\gamma)] \mathrm{P}\left[A\left(\gamma^{\prime}\right)\right]\left(d / a^{\prime}\right)^{j_{0}}\left(\frac{3 d}{a^{\prime}(2 d-7)}\right)^{j_{2}}
$$

We note that the exact value of $a^{\prime}$ is not important for our application, and the key point is that $d / a^{\prime}<d$ and that for $d \geq 8$, we have that $\frac{3 d}{a^{\prime}(2 d-7)}<d / 3<p^{-1}$, where in the notation of Lemmata 4.4 and $4.5, p:=\mathrm{P}\left[d\left(S_{i+1}, S_{i+1}^{\prime}\right)=2 \mid d\left(S_{i}, S_{i}^{\prime}\right)=\right.$ $2]$.

Proof of Proposition 4.8: For simplicity, assume that both $j_{0}$ and $j_{2}$ are divisible by 2 , in which case we can take $a^{\prime}=a$. The other cases are treated in an analogous manner and the possible small difference in the probabilities between the cases can be absorbed by taking some sufficiently smaller $a^{\prime}$ such that $1<a^{\prime}<a$.

We first observe that by construction (namely, by (4.15)) if $j_{0}+j_{2}+1 \leq 2 j-1$, then $C_{2 j-1} \cap C_{2 j}=A_{j}\left(\gamma^{\prime}\right)$. Denote,

$$
D_{0}:=\bigcap_{j=1}^{j_{0}} C_{j}, D_{2}:=\bigcap_{j=j_{0}+1}^{j_{0}+j_{2}} C_{j} \text { and } D_{\geq 4}:=\bigcap_{j=j_{0}+j_{2}+1}^{2 k-1} C_{j}
$$


We argue that the events $A(\gamma) \cap D_{2}$ and $D_{\geq 4}$ are independent. To see this, first note that $d\left(\left\{v_{i}^{\prime}: j_{0}+j_{2}+1 \leq i \leq 2 k-1\right\}, \gamma\right) \geq 3$. From the definition of $K_{j}$ in the case that $d\left(v_{j}, v_{j}^{\prime}\right)=2$, the event $D_{2}$ does not depend on the ages of the vertices in $\left\{v_{i}^{\prime}: j_{0}+j_{2}+1 \leq i \leq 2 k-1\right\}$ and also does not depend on the ages of any of their neighbors, apart from $v_{j_{0}+j_{2}}^{\prime}$, whose age is irrelevant for $D_{\geq 4}$.

Moreover, from the definition of $K_{i}$ when $d\left(v_{i}, v_{i}^{\prime}\right)=0, D_{0} \subset A(\gamma)$. Hence

$$
\mathrm{P}[A(\gamma) \cap C]=\mathrm{P}\left[A(\gamma) \cap D_{2} \cap D_{\geq 4}\right]=\mathrm{P}[A(\gamma)] \mathrm{P}\left[D_{2} \mid A(\gamma)\right] \mathrm{P}\left[D_{\geq 4}\right]
$$

Let $\left(X_{v_{j}^{\prime}}^{\prime}: j_{0}+1 \leq j \leq j_{0}+j_{2}\right)$ be i.i.d. Uniform $[0,1]$ random variables, independent of $\left(X_{v}: v \in \mathbb{Z}^{d}\right)$. For any $j_{0}+1 \leq j \leq j_{0}+j_{2}$, denote

$$
F_{j}\left(\gamma, \gamma^{\prime}\right)=F_{j}:=\left\{u \sim v_{j}^{\prime}: u \notin \gamma^{\prime} \cup N_{j}, u \nsim\left(\gamma^{\prime} \backslash\left\{v_{j}^{\prime}\right\}\right)\right\}
$$

(where the set $N_{j}$ is defined in (4.14)) and set

$$
K_{j}^{\prime}:=\left|\left\{u \in F_{j}: X_{u}<X_{v_{j}^{\prime}}^{\prime}\right\}\right| .
$$

Define

$$
B_{j}:=\left\{K_{j}^{\prime} \leq 2\right\} \text { and set } B:=\bigcap_{j=j_{0}+1}^{j_{0}+j_{2}} B_{j} .
$$

Note that by construction $\left|F_{j}\right| \geq 2 d-8$, for any $j_{0}+1 \leq j \leq j_{0}+j_{2}$. Moreover, by construction, the sets $F_{j_{0}+1}, \ldots, F_{j_{0}+j_{2}}$ are disjoint and are also disjoint from the set of vertices that the event $A(\gamma)$ depends on their ages. Using this and Proposition 4.7 (ii) we get that

$$
\mathrm{P}\left[D_{2} \mid A(\gamma)\right] \leq \mathrm{P}[B \mid A(\gamma)] \leq\left(\frac{3}{2 d-7}\right)^{j_{2}}
$$

Denote

$$
A_{0}:=\bigcap_{j \in\left[j_{0} / 2\right]} A_{j}\left(\gamma^{\prime}\right), A_{2}:=\bigcap_{j \in\left[\frac{j_{0}}{2}+1\right]}^{\frac{j_{0}+j_{2}}{2}} A_{j}\left(\gamma^{\prime}\right) \text { and } A_{\geq 4}:=\bigcap_{j \in\left[\frac{j_{0}}{2}+1\right]}^{\frac{j_{0}+j_{2}}{2}} A_{j}\left(\gamma^{\prime}\right) .
$$

Note that $A_{\geq 4}=D_{\geq 4}$. By Proposition 4.6, $\mathrm{P}\left[A_{0}\right] \geq(a / d)^{j_{0}}, \mathrm{P}\left[A_{2}\right] \geq(a / d)^{j_{2}}$. By (4.10) the events $A_{0}, A_{2}$ and $A_{\geq 4}$ are positively correlated. Thus by (4.17) and (4.18) we get that

$$
\begin{aligned}
\mathrm{P}[A(\gamma) \cap C] & \leq \mathrm{P}[A(\gamma)]\left(\frac{3}{2 d-7}\right)^{j_{2}} \mathrm{P}\left[D_{\geq 4}\right] \\
& \leq \mathrm{P}[A(\gamma)] \mathrm{P}\left[A_{0}\right](d / a)^{j_{0}} \mathrm{P}\left[A_{2}\right]\left(a^{-1} d\right)^{j_{2}}\left(\frac{3}{2 d-7}\right)^{j_{2}} \mathrm{P}\left[A_{\geq 4}\right] \\
& \leq \mathrm{P}[A(\gamma)] \mathrm{P}\left[A\left(\gamma^{\prime}\right)\right](d / a)^{j_{0}}\left(\frac{3 d}{a(2 d-7)}\right)^{j_{2}} \cdot \square
\end{aligned}
$$

Denote the uniform distribution on $\Gamma_{2 k-1}^{\operatorname{mon}}$ by $\nu_{2 k-1}$. Denote the expectation operator with respect to $\nu_{2 k-1} \times \nu_{2 k-1}$ by $\mathbf{E}_{2 k-1} \times \mathbf{E}_{2 k-1}$. Pick two paths $\gamma=\left(v_{1}, \ldots, v_{2 k}\right)$ and $\gamma^{\prime}=\left(v_{1}^{\prime}, \ldots, v_{2 k}^{\prime}\right)$ in $\Gamma_{2 k-1}^{\text {mon }}$ according to $\nu_{2 k-1} \times \nu_{2 k-1}$, where the choice of the 
paths is done independently of the layers model, that is, independently of the ages of the vertices. Recall the definition of $Z_{k}$ in (4.11). Observe that

$$
\begin{aligned}
\mathbf{E}\left[Z_{k}^{2}\right] & =\left|\Gamma_{2 k-1}^{\text {mon }}\right|^{-2} \sum_{\gamma, \gamma^{\prime} \in \Gamma_{2 k-1}} \frac{\mathrm{P}\left[A(\gamma) \cap A\left(\gamma^{\prime}\right)\right]}{\mathrm{P}[A(\gamma)] \mathrm{P}\left[A\left(\gamma^{\prime}\right)\right]}=\mathbf{E}_{2 k-1} \times \mathbf{E}_{2 k-1}\left[\frac{\mathrm{P}\left[A(\gamma) \cap A\left(\gamma^{\prime}\right)\right]}{\mathrm{P}[A(\gamma)] \mathrm{P}\left[A\left(\gamma^{\prime}\right)\right]}\right] \\
& \leq \mathbf{E}_{2 k-1} \times \mathbf{E}_{2 k-1}\left[\frac{\mathrm{P}\left[A(\gamma) \cap C\left(\gamma, \gamma^{\prime}\right)\right]}{\mathrm{P}[A(\gamma)] \mathrm{P}\left[A\left(\gamma^{\prime}\right)\right]}\right]=: \rho_{k},
\end{aligned}
$$

where the probability inside the expectation is taken with respect to the layers model for fixed $\gamma, \gamma^{\prime} \in \Gamma_{2 k-1}^{\mathrm{mon}}$ and the expectation outside indicates that we take an average according to random $\gamma, \gamma^{\prime}$ picked according to $\nu_{2 k-1} \times \nu_{2 k-1}$, independently of the layers model. By (4.13) and (4.19), we only need to find a constant $\beta>0$ such that for any $k, w_{k} \leq \beta$. We find such $\beta$ by combining Proposition 4.8 with Lemma 4.5 .

Let $\gamma=\left(v_{1}, \ldots, v_{2 k}\right)$ and $\gamma^{\prime}=\left(v_{1}^{\prime}, \ldots, v_{2 k}^{\prime}\right)$ be random paths in $\Gamma_{2 k-1}^{\text {mon }}$ chosen according to $\nu_{2 k-1} \times \nu_{2 k-1}$. We say that $\gamma$ and $\gamma^{\prime}$ are at distance $r$ at time $i$ if $d\left(v_{i}, v_{i}^{\prime}\right)=r$. We think about the paths as being exposed one vertex at a time according to the random walks from Definition 4.3. Notice that for any $i \in[2 k]$, $d\left(v_{i}, \gamma^{\prime}\right) \geq d\left(v_{i}, v_{i}^{\prime}\right)-1$. Whenever $v_{i-1} \neq v_{i-1}^{\prime}$ but $v_{i}=v_{i}^{\prime}, \gamma$ and $\gamma^{\prime}$ intersect each other for a random number of times (including time $i$ ) which is stochastically dominated by a Geometric $\left(1-d^{-1}\right)$ random variable.

Whenever the two paths are at distance 2 from each other, they have a chance of $\frac{3 d-4}{d^{2}}$ to stay at distance 2 in the next step. Thus, similarly to the previous case, whenever $d\left(v_{i-1}, v_{i-1}^{\prime}\right) \neq 2$, but $d\left(v_{i}, v_{i}^{\prime}\right)=2$, the two paths stay at distance 2 from each other for a random number of steps which is stochastically dominated by a Geometric $\left(1-3 d^{-1}\right)$ random variable. If $d\left(v_{i}, v_{i}^{\prime}\right)=2$, then the conditional probability that $v_{i+1}=v_{i+1}^{\prime}$, given that $d\left(v_{i+1}, v_{i+1}^{\prime}\right) \neq 2$ is by (4.7) (and the symmetry of the lattice)

$$
q_{2,0}:=\frac{d^{-2}}{1-(3 d-4) d^{-2}}=d^{-2}+O\left(d^{-3}\right) .
$$

Similarly, if $d\left(v_{i}, v_{i}^{\prime}\right)=2$ then the conditional probability that $d\left(v_{i+1}, v_{i+1}^{\prime}\right)=4$, given that $d\left(v_{i+1}, v_{i+1}^{\prime}\right) \neq 2$, is

$$
q_{2,4}:=1-d^{-2}-O\left(d^{-3}\right) .
$$

Once the paths reach distance 4 from each other, by (4.7) (and the symmetry of the lattice) the probability that the paths will ever be at distance 2 again is at most

$$
q_{4,2}:=4 d^{-2}+O\left(d^{-3}\right) .
$$

So the number of times the two paths will ever return from distance 4 to distance 2 is stochastically dominated by a Geometric $\left(1-4 d^{-2}-O\left(d^{-3}\right)\right)$ random variable. As long as the paths are at distance at least 4 , the events $A_{i}(\gamma)$ and $C_{2 i-i}\left(\gamma, \gamma^{\prime}\right) \cap$ $C_{2 i}\left(\gamma, \gamma^{\prime}\right)=A_{i}\left(\gamma^{\prime}\right)$ are independent. Hence, the only contributions to $\rho_{k}$ come from time intervals in which the paths stay at distance 0 from each other and from time intervals in which the paths stay at distance 2 from each other. To be more precise, one can extend Proposition 4.7 to cover also cases in which there may be several time intervals in which the paths are at distance 0 or 2 , where now $j_{0}$ and $j_{2}$ would be the total number of steps that the paths spend at distance 0 and 2 , respectively, from each other, so that (4.16) still holds. We omit the details. 
Let $s_{0}=s_{0}\left(\gamma, \gamma^{\prime}\right)$ and $s_{2}=s_{2}\left(\gamma, \gamma^{\prime}\right)$ be the number of time intervals in which the paths are at distance 0 or 2 from each other, respectively. Set

$$
\begin{aligned}
& p_{0}:=\sum_{j \geq 1}\left(d / a^{\prime}\right)^{j} d^{-j+1}=d \frac{1}{a^{\prime}-1}, \\
& p_{2}:=\sum_{j \geq 1}\left(\frac{3 d}{a^{\prime}(2 d-7)}\right)^{j}(3 / d)^{j-1}=\left(\frac{3 d}{a^{\prime}(2 d-7)}\right) \frac{1}{1-\frac{9}{a^{\prime}(2 d-7)}} .
\end{aligned}
$$

We now analyze the contribution to $\rho_{k}$ coming from the time intervals in which the paths are at distance 0 or 2 from each other, respectively. Again, we employ the random walk interpretation and think about $\gamma$ and $\gamma^{\prime}$ as being exposed one vertex at a time. By (4.16) and the aforementioned stochastic dominations by Geometric random variables, each time interval in which the paths are at distance 0 or 2 contributes to $\rho_{k}$ a multiplicative term of at most $p_{0}$ or $p_{2}$, respectively. Moreover, since in the case $k=\infty$, given $\left(s_{0}, s_{2}\right)$, the lengths of the different time intervals are independent, then given $\left(s_{0}, s_{2}\right)$, the total contribution of all the different time intervals is bounded by $p_{0}^{s_{0}} p_{2}^{s_{2}}$. Whence, in order to bound $w_{k}$ it suffices to bound $\mathbf{E}_{2 k-1} \times \mathbf{E}_{2 k-1}\left[p_{0}^{s_{0}\left(\gamma, \gamma^{\prime}\right)} p_{2}^{s_{2}\left(\gamma, \gamma^{\prime}\right)}\right]$.

Let $q_{2,0}, q_{2,4}, q_{4,2}$ be as above. Consider the following Markov chain with state space $\{0,2,4, \infty\}$, whose initial state is 0 . If the current state of the chain is 0 , then the next state is $2 \mathrm{w} . \mathrm{p} .1$. If the current state of the chain is 2 , then the next state is 0 w.p. $q_{2,0}=d^{-2}+O\left(d^{-3}\right)$ and otherwise (w.p. $\left.q_{2,4}\right)$ it is 4 . If the current state of the chain is 4 , then w.p. $q_{4, \infty}:=1-q_{4,2}=1-4 d^{-2}+O\left(d^{-3}\right)$ the chain moves to an absorbing state $\infty$ and otherwise (w.p. $q_{4,2}$ ) it moves to state 2 . Let $r_{0}, r_{2}$ be the number of times this chain visits states 0 and 2 , respectively, before getting absorbed in $\infty$. Note that for any $1 \leq k_{0} \leq k_{2} \in \mathbb{N}$

$$
\begin{aligned}
& \mathrm{P}\left[r_{0}=k_{0}, r_{2}=k_{2}\right]=q_{2,0}^{k_{0}-1} q_{4,2}^{k_{2}-k_{0}} q_{4, \infty} \\
& =\left[d^{-2}+O\left(d^{-3}\right)\right]^{k_{0}-1}\left[4 d^{-2}+O\left(d^{-3}\right)\right]^{k_{2}-k_{0}}\left[1-4 d^{-2}+O\left(d^{-3}\right)\right] .
\end{aligned}
$$

By the above analysis, $\left(r_{0}, r_{2}\right)$ stochastically dominates $\left(s_{0}\left(\gamma, \gamma^{\prime}\right), s_{2}\left(\gamma, \gamma^{\prime}\right)\right)$, when $\left(\gamma, \gamma^{\prime}\right)$ is picked according to $\nu_{2 k-1} \times \nu_{2 k-1}$. Let $d$ be sufficiently large so that

$p_{0}, p_{2}>1$. Using the aforementioned stochastic domination on the increasing function $f\left(x_{0}, x_{2}\right)=p_{0}^{x_{0}} p_{2}^{x_{2}}$ in conjunction with (4.20) and (4.21), we get that

$\rho_{k} \leq \sum_{k_{0}, k_{2}} p_{0}^{k_{0}} p_{2}^{k_{2}} \mathrm{P}\left[s_{0}=k_{0}, s_{2}=k_{2}\right] \leq \sum_{k_{0}, k_{2}} p_{0}^{k_{0}} p_{2}^{k_{2}} \mathrm{P}\left[r_{0}=k_{0}, r_{2}=k_{2}\right] \leq b p_{0} p_{2}=: \beta$

for some absolute constant $b$. This was noted earlier to imply (4.13), which concludes the proof by the paragraph following (4.13).

\section{The first 3 layers in random graphs}

In this section we prove Theorem 1.6. Our approach utilizes an auxiliary random graph, similar to an auxiliary construction considered in Feige et al. (2016).

5.1. Preliminaries. A connected component that contains a linear fraction of the vertices of a graph is commonly referred to as a giant component. There has been extensive work on the formation of giant components in random graph models and 
in percolation on random graphs (see for example Fountoulakis, 2007; Krivelevich and Sudakov, 2013; Janson, 2009; Molloy and Reed, 1995).

We now state a few basic facts about random graphs with a given degree sequence. Let $\bar{d}$ be a non-increasing sequence of $n$ nonnegative integers and let $d_{i}$ be the $i$ th element of $\bar{d}$. Throughout, we assume that $\sum d_{i}$ is even and that $d_{1} \leq d$ for some $d$ which does not depend on $n$.

We consider the random graph model $\mathcal{G}_{n, \bar{d}}$ in which a graph is sampled according to the uniform distribution over all graphs labeled by the set $[n]$ in which vertex $i$ has degree $d_{i}$. We indicate that a random graph $G$ has such a distribution by writing $G \sim \mathcal{G}_{n, \bar{d}}$. If $G \sim \mathcal{G}_{n, \bar{d}}$ we say that $G$ is a random graph with a given degree sequence $\bar{d}$. In practice, we consider a sequence of degree sequences $\bar{d}(n)$, where $\bar{d}(n)=\left(d_{1}(n), \ldots, d_{k_{n}}(n)\right)$, and a sequence of graphs $G_{n} \sim \mathcal{G}_{k_{n}, \bar{d}(n)}$, where $\lim _{n \rightarrow \infty} k_{n}=\infty$. We hide the dependence on $n$ from our notation.

An intimately related model to $\mathcal{G}_{n, \bar{d}}$ is the configuration model $\mathcal{P}_{n, \bar{d}}$, which is a model for generating a random multigraph whose vertex set is labeled by $[n]$ and vertex $i$ has $d_{i}$ "half-edges". The half-edges are combined into edges by choosing uniformly at random a matching of all "half-edges". We indicate that a random multigraph $G$ has such a distribution by writing $G \sim \mathcal{P}_{n, \bar{d}}$.

Given a multigraph sampled according to the configuration model, Molloy and Reed (1995) provide a criterion for the existence of a giant component. We refer to the condition in the Theorem 5.1 as the Molloy-Reed condition. The exact statement of their result involves some technical conditions and parameters that are omitted here due to our bounded degree setup.

Theorem 5.1. Let $(\bar{d}(n): n \in \mathbb{N})$ be a sequence of degree sequences. Assume that $\sup _{n} d_{1}(n)<\infty$. Denote by $\lambda_{i}(n)$ the fraction of vertices of degree $i$ in $\bar{d}(n)$. Let

$$
Q(\bar{d}(n))=\sum_{i \geq 1} \lambda_{i}(n) i(i-2) .
$$

Assume further that $Q(\bar{d}(n))>\epsilon>0$ for all sufficiently large $n$. Let $G_{n} \sim \mathcal{P}_{n, \bar{d}(n)}$. Then the probability of $G_{n}$ not having a giant component is exponentially small in $n$.

We now state a few elementary facts about $\mathcal{G}_{n, \bar{d}}$ and $\mathcal{P}_{n, \bar{d}}$ and their relations (see e.g. Wormald, 1999 Theorem 2.6). We say that a set of variables $X_{i}^{(n)}$ for $i$ in some finite set $I$, defined on a sequence of probability spaces indexed by $n$ are asymptotically independent Poisson with means $\lambda_{i}$ if their joint distribution tends to that of independent Poisson variables whose means are fixed numbers $\lambda_{i}$. When $\bar{d}=(d, d, \ldots, d)$ is the fixed sequence, we write $\mathcal{G}_{n, d}$ and $\mathcal{P}_{n, d}$ instead of $\mathcal{G}_{n, \bar{d}}$ and $\mathcal{P}_{n, \bar{d}}$, respectively.

Theorem 5.2. $\quad$ (i) Let $d \geq 3$. Let $X_{i}^{(n)}$ be the number of cycles of length $i$ in $G_{n, d} \sim \mathcal{P}_{n, d}$. Then $X_{1}^{(n)}, \ldots, X_{k}^{(n)}$ are asymptotically independent Poisson with means $\lambda_{i}:=(d-1)^{i} / 2 i$, for all $k \geq 1$.

(ii) Let $\bar{d}=\left(d_{1}, \ldots, d_{n}\right)$ be a degree sequence with $d_{1} \leq d$. Let $Y_{i}^{(n)}$ be the number of cycles of length $i$ in $G_{n, \bar{d}} \sim \mathcal{P}_{n, \bar{d}}$. Then for all $n$ and $k \geq 1$, the joint distribution of $Y_{1}^{(n)}, \ldots, Y_{k}^{(n)}$ is stochastically dominated by that of $X_{1}^{(n)}, \ldots, X_{k}^{(n)}$ from part (i). 
(iii) Let $G_{n, \bar{d}} \sim \mathcal{P}_{n, \bar{d}}$. Let $Y_{i}^{(n)}$ be as in (ii). Given $Y_{1}=Y_{2}=0$, we have that $G_{n, \bar{d}} \sim \mathcal{G}_{n, \bar{d}}$.

5.2. Proof of Theorem 1.6. Proof: Fix some odd $k \geq 15$ to be determined later. Let $G=([n], E) \sim \mathcal{G}_{n, \bar{d}}$ for some degree sequence $\bar{d}:=\left(d_{1}, \ldots, d_{n}\right)$ with $d_{1} \leq d$. We first argue that with probability $1-\exp (-\Omega(n))$ there are less than $\frac{n}{4 d^{k+2}}$ vertices belonging to a cycle of length at most $k+2$ in $G$. Call this event $F$. By part (iii) of Theorem 5.2 it suffices to show that the same holds for $H^{\prime} \sim \mathcal{P}_{n, \bar{d}}$. By Theorem 5.2 the expected number of vertices belonging to a cycle of length at most $k+2$ is $O(1)$. Consider the exposure process of $H^{\prime}$ in which the half edges are exposed one at a time. Consider the Doob's martingale of the number of vertices belonging to a cycle of length at most $k+2$ w.r.t. this exposure procedure. The increments of this martingale can be bounded by $d^{k+2}$. Indeed, using the description of the configuration model, the increments can be bounded by the maximal change in the number of vertices belonging to a cycle of length at most $k+2$ possible by the operation of adding a single edge to a graph with degree sequence $\bar{d}$. Indeed, to control the increments, after exposing a certain edge by matching together its two half-edges, we can now increase the number of half-edges at the corresponding vertices by one (or 2 if the exposed edge was a self loop) and the distribution of the remaining edges will remain unaffected. This idea can be used for bounding the increments both from above and below (we leave the details as an exercise). Hence, using Azuma inequality we obtain the desired bound.

On the event $F$, there exist a constant $c=c(d)$ and a set $I \subset[n]$ with $|I| \geq c n$ such that every two vertices in $I$ are of distance at least 15 from one another and the ball of radius $k+2$ around each of them does not contain a cycle. Thus on the event $F$, there are two sets $A, B$ both of size $\lceil c n\rceil$ such that every vertex in $A$ is adjacent to exactly one vertex in $B$ (and vice versa) and the ball of radius $k+1$ around every vertex in $A \cup B$ does not contain a cycle.

Assuming the event $F$ occurs, fix such sets $A, B$. Consider the graph $G^{\prime}$ obtained by removing from $G$ all the edges between $A$ and $B$. Denote the set of deleted edges by $M$. Note that given $G^{\prime}$ and the set $D:=A \cup B$, the conditional distribution of $M$ is uniformly distributed among all perfect matchings of the set $D$ (we assume $G, A$ and $B$ are unknown and only $G^{\prime}$ and $D=A \cup B$ were exposed).

Consider the vertex-induced random subgraph of $G^{\prime}$ (induced w.r.t. $G^{\prime}$ ) on the vertex set

$$
\left\{u \in[n] \backslash D: u \in T_{3}\left(G^{\prime}\right)\right\} \cup\left\{u \in D: u \in T_{2}\left(G^{\prime}\right)\right\} \text {, denoted by } R .
$$

We use the same source of randomness to generate the ages of the vertices in $T_{3}(G)$ and in $T_{3}\left(G^{\prime}\right)$. Note that for $u \in[n] \backslash D$, we have that $u \in T_{3}\left(G^{\prime}\right)$ iff $u \in T_{3}(G)$ as the set of neighbors of $u$ in $G$ is the same as the set of its neighbors in $G^{\prime}$. Also, note that since for every $u \in D$ the set of neighbors of $u$ in $G^{\prime}$ is contained in the set of its neighbors in $G$ and is smaller by one neighbor. Hence if $u \in T_{2}\left(G^{\prime}\right)$, then it must be the case that $u \in T_{3}(G)$.

Consider the following (random) set of edges $M^{\prime}:=\{\{v, u\} \in M: v, u \in R\}$. The requirement $\{v, u\} \in M$ implies that $v, u \in D$ and thus the requirement that $v, u \in R$ implies that they belong to $T_{2}\left(G^{\prime}\right)$. Whence $R \cup M^{\prime} \subset T_{3}(G)$, where by $R \cup M^{\prime}$ we mean the graph obtained by adding the edges of $M^{\prime}$ to $R$. Hence it suffices to show that $R \cup M^{\prime}$ has a giant component with probability $1-\exp (-\Omega(n))$. 
For every $u \in D$ let $C_{u}^{\prime}$ be the intersection of the connected component of $u$ in $R$ with the set $\left\{v: d_{G^{\prime}}(u, v) \leq k\right\}$. Notice that for every $u \in D$, we have that $C_{u}^{\prime}$ depends only on the ages of the vertices of distance at most $k+1$ from $u$ (distance w.r.t. the graph $G^{\prime}$ ). Since (by construction) up to a distance of $k+1$ from any $u \in D$ the graph looks like a tree, by Lemma 2.8 there exist some constants $\bar{c}>0$ and $a>1$ (both independent of $k$ ) such that every $u \in D$ satisfies $\left|C_{u}^{\prime}\right| \geq a^{k}$ with probability at least $\bar{c} d^{-1}$. We call a vertex $u \in D$ which satisfies $\left|C_{u}^{\prime}\right| \geq a^{k}$ good. The occurrence or non-occurrence of the event that a vertex $v \in D$ is good can affect the conditional probability of the event that $u \in D$ is good only for those $u \in D$ such that $d_{G^{\prime}}(v, u) \leq 3 k$. Denote the set of good vertices by Good $(\subset D)$. Let $D(\ell)$ be the event that $\mid$ Good $\mid \geq \ell$. By the assumption that the bound on the maximal degree, $d$, is a constant, Azuma inequality (Theorem 6.1) implies that for some constant $c^{\prime}=c^{\prime}(d)>0$ (independent of $k$ )

$$
\mathrm{P}\left[D\left(c^{\prime} n\right) \mid F\right] \geq 1-\exp [-\Omega(n)] .
$$

On the event $D\left(c^{\prime} n\right) \cap F$ we have a collection of at most $n / a^{k}$ connected components of $R$ each of size at least $a^{k}$ and the union of these connected components contains at least $c^{\prime} n$ vertices of $D$. If a connected component is of size greater than $a^{k}$, then in what comes we artificially treat it as several distinct connected components each of size between $a^{k}$ and $2 a^{k}$ by partitioning it in a arbitrary manner into (disjoint) sets of sizes between $a^{k}$ and $2 a^{k}$. Call the collection of all "large" components of $R$ we obtain in this manner $L$ (where we say a component is "large" if its size is between $a^{k}$ and $\left.2 a^{k}\right)$. Note that

$$
c^{\prime} n a^{-k} \leq|L| \leq n a^{-k}
$$

Construct now an auxiliary (random) multigraph $H$ with two sets of vertices, $U_{1}$ and $U_{2}$. Every component in $L$ serves as a vertex in $U_{1}$, and the number of good $D$ vertices in a component serves as the number of half-edges of the corresponding $U_{1}$-vertex (to avoid confusion we shall refer to the vertices in $U_{1}$ as super-vertices (following the terminology of Feige et al., 2016). Hence, on $D\left(c^{\prime} n\right) \cap F$, we have that $c^{\prime} n / a^{k} \leq\left|U_{1}\right| \leq n / a^{k}$ and as every good vertex in $D$ within a component in $L$ contributes one edge to the degree of the super-vertex of $H$ corresponding to its component in $T_{3}\left(G^{\prime}\right)$, we get that

$$
\sum_{u \in U_{1}} d_{u} \geq c^{\prime} n .
$$

The set $U_{2}$ consists of all the non-good vertices in $D$. Every vertex in $U_{2}$ has degree 1.

Consider now the configuration model for generating random multigraphs with vertex set and number of half-edges for each vertex as described above and call the resulting random multigraph $H$. The distribution of $G$ given $G^{\prime}$ and $D$ (conditioned on the event $D\left(c^{\prime} n\right) \cap F$ whose probability is $\left.1-\exp (-\Omega(n))\right)$ can be coupled with $H$ in a natural manner. Since the number of vertices of $H$ (on the event $D\left(c^{\prime} n\right) \cap F$ ) is $\Omega(n)$, if $H$ contains a giant component then so does $T_{3}(G)$. Namely, a giant component in $H$ of size at least $d \alpha n$ implies a giant component in $T_{3}(G)$ of size at least $\alpha n$.

To determine that $H$ is likely to have a giant component we use Theorem 5.1. We first need the following proposition taken from Feige et al. (2016). 
Proposition 5.3. Consider two vertices of degree $r$ and $r^{\prime} \geq r+2$. Then the expression $\sum_{i>1} \alpha_{i} d_{i}\left(d_{i}-2\right)>0$ decreases by replacing them by vertices of degrees $r+1$ and $r^{\prime}-1$.

Proof: Initially the contribution of the two vertices is $r(r-2)+r^{\prime}\left(r^{\prime}-2\right)$. After replacement it is $(r+1)(r-1)+\left(r^{\prime}-1\right)\left(r^{\prime}-3\right)$, which is smaller by $2\left(r^{\prime}-r-1\right)$.

Observe that on $D\left(c^{\prime} n\right) \cap F$, by (5.2) and (5.3) the average degree of a supervertex in $U_{1}$ is at least $c_{1} a^{k}$ and the fraction of $U_{1}$ vertices in $H$ is at least $c_{2} a^{-k}$ for some constants $c_{1}, c_{2}>0$ independent of $k$. Let $\ell(k):=\left\lfloor c_{1} a^{k}\right\rfloor$. We denote the degree sequence of $H=\left(V^{\prime}, E^{\prime}\right)$ by $d_{1}(H), \ldots, d_{\left|V^{\prime}\right|}(H)$. The above proposition, together with the fact that the expression $\sum_{i \geq 0} \alpha_{i} d_{i}(H)\left(d_{i}(H)-2\right)$ is a monotone function of the $d_{i}(H)$ 's, implies that for the graph $H$, the expression $\sum_{i>0} \alpha_{i} d_{i}(H)\left(d_{i}(H)-2\right)$ is minimized (on $D\left(c^{\prime} n\right) \cap F$ ) when all vertices of $U_{1}$ are of degree $\ell(k)$. The fraction of $U_{2}$ vertices of $H$ can be bounded from above by 1 . The Molloy-Reed condition is indeed satisfied whenever $k$ is sufficiently large, as

$$
\sum_{i \geq 0} \alpha_{i} d_{i}(H)\left(d_{i}(H)-2\right) \geq 1 \cdot(-1)+c_{2} a^{-k} \ell(k)(\ell(k)-2)>\epsilon,
$$

for some constant $\epsilon>0$. So if we fix such $k$ we have that the Molloy-Reed condition indeed holds for $H$.

5.3. Possible extensions. We now discuss some technicalities related to possible relaxations of the assumptions on the degree sequence in Theorem 1.6. We then discuss the connection of Theorem 1.6 (and the aforementioned relaxations) to the analysis of $T_{3}(G(n, c / n))$. We end by discussing an alternative approach that can cover the more general case of large girth expanders and some of the difficulties related to that approach.

The assumption in Theorem 1.6 that the minimal degree is at least 3 could be relaxed to allow degree 2 vertices and is present mostly for convenience. To see this, observe that the existence of degree 2 vertices (as long as we also have some fraction of vertices of strictly larger degree) in our random graphs setup, would still allow one to get a parallel version of Lemma 2.8 with smaller $a>1$ and larger $k$. The assumption that there are no degree 1 vertices is more substantial, though with some work the proof can be adapted to the situation where the fraction of such vertices is sufficiently small, although this would complicate manners substantially. The difficulty would then be showing that a parallel statement to Lemma 2.8 still holds in such a setup.

Hence it is reasonable to expect that if $c>1$ is sufficiently large and $G \sim G\left(n, \frac{c}{n}\right)$ (i.e. $G$ is an Erdös-Rényi random graph with parameter $c / n$ ), then $T_{3}(G)$ has a giant component w.h.p.. Note that $G$ would not be of bounded degree, but this turns out to not be a major difficulty (for sufficiently large $d=d(c)$ and $k=k(c) \in \mathbb{N}$, the number of vertices which would not have a vertex of degree more than $d$ in a ball of radius $k$ around them in $G$ is w.h.p. linear in $n)$. We know that when $c-1$ is positive but sufficiently small, then w.h.p. all components of $T_{3}(G)$ are of size $O(\log n)$. Whence one should expect a phase transition with respect to $c$ for the existence of a giant component in $T_{3}(G)$. It is left as an open problem to verify this and to find the critical $c$. We note that a-priori it is not clear that there is monotonicity w.r.t. $c$ for the existence of a giant component w.h.p. in $T_{3}(G)$, but we believe this is indeed the case. 
Question 5.4. Let $G \sim G(n, c / n)$. What is the critical $c$ for the existence of a giant component in $T_{3}(G)$ ?

\section{Acknowledgements}

The author would like to thank Itai Benjamini, Uriel Feige, Daniel Reichman and Allan Sly for many useful discussions.

\section{Appendix}

In this section we state a few theorems that where used in this work. We start with Azuma inequality (see e.g. Theorem 13.2 in Lyons and Peres, 2016).

Lemma 6.1 (Azuma inequality). Let $X_{0}, \ldots, X_{n}$ be a martingale such that for every $1 \leq k<n$ it holds that $\left|X_{k}-X_{k-1}\right| \leq c_{k}$. Then for every nonnegative integer $t$ and real $B>0$

$$
\mathrm{P}\left(X_{t}-X_{0} \leq-B\right) \leq \exp \left(\frac{-B^{2}}{\sum_{i=1}^{t} c_{i}^{2}}\right) .
$$

The following Theorem is due to Paley and Zygmund (see e.g. Lyons and Peres, 2016 pg 162)

Theorem 6.2 (Paley and Zygmund's inequality). If $X$ is a random variable with mean 1 and $0<t<1$, then

$$
\mathrm{P}(X>t) \geq \frac{(1-t)^{2}}{\mathbf{E}\left[X^{2}\right]} .
$$

Suppose that we have a countable (possibly finite) set of vertices, $S$, and a state space $\Omega:=\mathbb{R}^{S}$, with the product topology and the corresponding Borel $\sigma$-algebra $\mathcal{B}$. We now define the notion of stochastic domination. Given $\omega_{1}, \omega_{2} \in \Omega$, we say that $\omega_{1} \leqslant \omega_{2}$, whenever $\omega_{1}(s) \leqslant \omega_{2}(s)$ for any $s \in S$. We say that a measurable function $f: \Omega \rightarrow \mathbb{R}$ is increasing, whenever $\omega_{1} \leqslant \omega_{2} \in \Omega$ implies $f\left(\omega_{1}\right) \leq f\left(\omega_{2}\right)$. When we have real valued random variables $X=\left(X_{s}: s \in S\right)$, we sometimes say an event is increasing with respect to $X$. By this we mean that the indicator function of that event may be written as $f(X)$ for some increasing function $f: \Omega \rightarrow \mathbb{R}$. We say it is decreasing when $-f$ is increasing. Given two Borel probability measures on $\Omega, \mu$ and $\nu$, we say that $\mu$ stochastically dominates $\nu$, and denote this by $\mu \geqslant \nu$, if for any continuous increasing function $f$ we have

$$
\int_{\Omega} f d \mu \geq \int_{\Omega} f d \nu
$$

We say that an event is increasing (respectively, decreasing) if its indicator function is increasing (respectively, decreasing). It is not hard to prove that it suffices to restrict to the case that $f$ is an indicator of an increasing event. Moreover, by a simple limiting argument, it suffices to consider increasing indicators of events that depends only on finitely many co-ordinates.

The following Theorem is due to Harris (1960). For a proof, see e.g. Section 2.2. of Grimmett (1999). 
Theorem 6.3 (Harris/FKG inequality). Suppose that we have a countable (possibly finite) set of vertices, $S$, and a state space $\Omega=\mathbb{R}^{S}$. Assume we have independent $\mathbb{R}$ valued random variables $X:=\left(X_{s}: s \in S\right)$. Let $f, g: \Omega \rightarrow \mathbb{R}$ be increasing, then

$$
\mathbf{E}[f(X) g(X)] \geq \mathbf{E}[f(X)] \mathbf{E}[g(X)] .
$$

The same holds when both $f$ and $g$ are decreasing. If $f$ is increasing and $g$ is decreasing the inequality holds in the reverse direction.

We now present a more general Theorem we shall need. Let $k \in \mathbb{N}$. For any $x, y \in \mathbb{R}^{k}$ denote by $x \wedge y$ and $x \vee y$ their coordinate-wise minimum and maximum, respectively. A set $S \subset \mathbb{R}^{k}$ is called a sub-lattice if whenever $x, y \in S$ so are $x \wedge y$ and $x \vee y$.

Theorem 6.4. Let $X:=\left(X_{1}, \ldots, X_{k}\right)$ be independent real valued random variables. Let $S \subset \mathbb{R}^{k}$ be a sub-lattice so that $\mathrm{P}[X \in S]>0$. Let $A, B$ be two increasing events with respect to $X$. Then,

$$
\mathrm{P}[A \cap B \mid X \in S] \geq \mathrm{P}[A \mid X \in S] \mathrm{P}[B \mid X \in S] .
$$

Random variables that satisfy (6.1) are called affiliated. It is well-known (e.g. the appendix of Milgrom and Weber, 1982$)$ that if $\left(X_{1}, \ldots, X_{k}\right)$ have a joint density function $f$ which satisfies

$$
f(x \vee y) f(x \wedge y) \geq f(x) f(y),
$$

for Lebesgue a.e. $(x, y) \in \mathbb{R}^{2 k}$, then $\left(X_{1}, \ldots, X_{k}\right)$ are affiliated. We call such a nonnegative function satisfying (6.2) affiliated. It is easy to verify that if $f\left(z_{1}, z_{2}\right)=$ $g\left(z_{1}\right) h\left(z_{2}\right)$ and $g$ and $h$ are affiliated, then so is $f$. Since any non-negative $g: \mathbb{R} \rightarrow \mathbb{R}$ is trivially affiliated and a joint density function of independent random variables factors to a product of the marginal densities, we indeed get that independent random variables are always affiliated as stated in Theorem 6.4.

\section{References}

I. Benjamini, A. Nachmias and Y. Peres. Is the critical percolation probability local? Probab. Theory Related Fields 149 (1-2), 261-269 (2011). MR2773031.

I. Benjamini, R. Pemantle and Y. Peres. Unpredictable paths and percolation. Ann. Probab. 26 (3), 1198-1211 (1998). MR1634419.

J. T. Cox and R. Durrett. Oriented percolation in dimensions $d \geq 4$ : bounds and asymptotic formulas. Math. Proc. Cambridge Philos. Soc. 93 (1), 151-162 (1983). MR684285.

U. Feige, J. Hermon and D. Reichman. On giant components and treewidth in the layers model. Random Structures Algorithms 48 (3), 524-545 (2016). MR3481272.

U. Feige and D. Reichman. Recoverable values for independent sets. Random Structures Algorithms 46 (1), 142-159 (2015). MR3291296.

W. Feller. An introduction to probability theory and its applications. Vol. II. Second edition. John Wiley \& Sons, Inc., New York-London-Sydney (1971). MR0270403.

N. Fountoulakis. Percolation on sparse random graphs with given degree sequence. Internet Math. 4 (4), 329-356 (2007). MR2522948.

G. Grimmett. Percolation, volume 321 of Grundlehren der Mathematischen Wissenschaften [Fundamental Principles of Mathematical Sciences]. Springer-Verlag, Berlin, second edition (1999). ISBN 3-540-64902-6. MR1707339. 
S. Janson. On percolation in random graphs with given vertex degrees. Electron. J. Probab. 14, no. 5, 87-118 (2009). MR2471661.

M. Krivelevich and B. Sudakov. The phase transition in random graphs: a simple proof. Random Structures Algorithms 43 (2), 131-138 (2013). MR3085765.

R. Lyons. The Ising model and percolation on trees and tree-like graphs. Comm. Math. Phys. 125 (2), 337-353 (1989). MR1016874.

R. Lyons. Random walks, capacity and percolation on trees. Ann. Probab. 20 (4), 2043-2088 (1992). MR1188053.

R. Lyons and Y. Peres. Probability on trees and networks, volume 42 of Cambridge Series in Statistical and Probabilistic Mathematics. Cambridge University Press, New York (2016). ISBN 978-1-107-16015-6. MR3616205.

Paul R. Milgrom and Robert J. Weber. A theory of auctions and competitive bidding. Econometrica: Journal of the Econometric Society pages 1089-1122 (1982).

M. Molloy and B. Reed. A critical point for random graphs with a given degree sequence. In Proceedings of the Sixth International Seminar on Random Graphs and Probabilistic Methods in Combinatorics and Computer Science, "Random Graphs '93" (Poznań, 1993), volume 6, pages 161-179 (1995). MR1370952.

D. Reichman. New bounds for contagious sets. Discrete Math. 312 (10), 1812-1814 (2012). MR2901177.

N. C. Wormald. Models of random regular graphs. In Surveys in combinatorics, 1999 (Canterbury), volume 267 of London Math. Soc. Lecture Note Ser., pages 239-298. Cambridge Univ. Press, Cambridge (1999). MR1725006. 